\title{
Quantitative proteomics identifies PTP1B as modulator of $B$ cell antigen receptor signaling
}

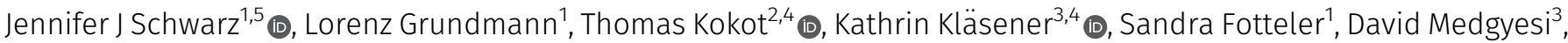 \\ Maja Köhn ${ }^{2,4,5}$ (D), Michael Reth ${ }^{3,4,5}$ (D), Bettina Warscheid ${ }^{1,4,5}$ (D)
}

\begin{abstract}
B cell antigen receptor (BCR) signaling is initiated by protein kinases and limited by counteracting phosphatases that currently are less well studied in their regulation of $B C R$ signaling. Here, we used the B cell line Ramos to identify and quantify human B cell signaling components. Specifically, a protein tyrosine phosphatase profiling revealed a high expression of the protein tyrosine phosphatase 1B (PTP1B) in Ramos and human naive B cells. The loss of PTP1B leads to increased $B$ cell activation. Through substrate trapping in combination with quantitative mass spectrometry, we identified 22 putative substrates or interactors of PTP1B. We validated Ig $\alpha, \mathrm{CD} 22, \mathrm{PLC} \gamma 1 / 2, \mathrm{CBL}$, BCAP, and APLP2 as specific substrates of PTP1B in Ramos B cells. The tyrosine kinase BTK and the two adaptor proteins GRB2 and VAV1 were identified as direct binding partners and potential substrates of PTP1B. We showed that PTP1B dephosphorylates the inhibitory receptor protein CD22 at phosphotyrosine 807. We conclude that PTP1B negatively modulates BCR signaling by dephosphorylating distinct phosphotyrosines in B cell-specific receptor proteins and various downstream signaling components.
\end{abstract}

DOI 10.26508/lsa.202101084 | Received 2 April 2021 | Revised 28 August 2021 | Accepted 1 September 2021 | Published online 15 September 2021

\section{Introduction}

Protein tyrosine phosphorylation plays a crucial role in the regulation of signal transduction processes and a tight regulation is crucial for cell fate decisions (Hunter, 2000). Over the years, knowledge about protein tyrosine kinases (PTKs) has increased considerably, whereas information about protein tyrosine phosphatases (PTPS) and their role in the initiation or termination of signaling is still incomplete. The human genome encodes 109 PTPS, which belong to three different enzyme classes (Damle \& Köhn,
2019). The vast majority of those belong to class I, which is defined by their signature motif $\mathrm{HC}(\mathrm{X})_{5} \mathrm{R}$ (Li et al, 2013). To this class belong the "classical PTPS," consisting of both receptor type and nonreceptor type phosphatases (Alonso et al, 2004). In general, the substrate specificity of PTPs is defined by their catalytic domain in combination with domains for localization and substrate recruitment in a cellular context (Tiganis \& Bennett, 2007).

PTP1B is a classical, ubiquitously expressed non-receptor type phosphatase comprised of an $\mathrm{N}$-terminal catalytic domain, two proline-rich motifs and an ER-targeting domain in its C-terminal region (Yip et al, 2010). The active site of PTP1B contains the catalytic cysteine residue which is needed for the dephosphorylation of substrate tyrosine residues. The two proline-rich motifs enable the binding of src-homology 3 (SH3) domain-containing proteins, such as p130Cas (Liu et al, 1996). Notably, PTP1B localizes to the ER membrane, where the C-terminal short tail enters the ER lumen (Frangioni et al, 1992), whereas both the proline-rich motifs and the catalytic domain are residing in the cytosol. Known substrates of PTP1B include several receptor tyrosine kinases (RTKS) and receptor-associated kinases including the insulin receptor, the epidermal- (EGFR), platelet-derived- (PDGFR), and insulin-like growth factor receptor (IGFR) as well as JAK2 in various nonhematopoietic cell types (Flint et al, 1997; Elchebly et al, 1999; Myers et al, 2001; Haj et al, 2003; Fan et al, 2013).

PTP1B is also expressed in the hematopoietic cell linage (Lu et al, 2008; Xu et al, 2008; Martin-Granados et al, 2015). Mice which are deficient for both PTP1B and the tumor suppressor p53 have an accumulation of $B$ cells in their bone marrow and lymph nodes, which leads to an increased susceptibility for B cell lymphomas (Dubé et al, 2005). Furthermore, mice with a B cell-specific deletion of PTP1B develop systemic autoimmunity as they age, with elevated anti-double stranded DNA antibodies in their sera, spontaneous germinal centre formation, and glomerulonephritis (Medgyesi et al, 2014).

${ }^{1}$ Biochemistry and Functional Proteomics, Institute of Biology II, Faculty of Biology, University of Freiburg, Freiburg, Germany ${ }^{2}$ Integrative Signalling Research, Institute of Biology III, Faculty of Biology, University of Freiburg, Freiburg, Germany ${ }^{3}$ Department for Molecular Immunology, Institute of Biology III, Faculty of Biology, University of Freiburg, Freiburg, Germany ${ }^{4}$ Signalling Research Centres BIOSS and CIBSS, University of Freiburg, Freiburg, Germany ${ }^{5}$ Spemann Graduate School of Biology and Medicine (SGBM), University of Freiburg, Freiburg, Germany 
Analysis of splenic B cells in these mice revealed p38 MAPK-pY182 as a specific substrate of PTP1B.

In this work, we studied the proteome of the human Burkitt's lymphoma B cell line Ramos at absolute quantitative scale. By comparing quantitative proteome and transcriptome data and by profiling the expression of classical PTPS in Ramos and naive $B$ cells, we revealed a role of PTP1B in signal transduction from the $B$ cell antigen receptor (BCR). Loss of PTP1B resulted in increased phosphorylation of several BCR signaling components. Through a quantitative in vivo substrate trapping approach, we identified 22 substrates and/or binding partners of PTP1B in human Ramos $B$ cells, many of which have B cell-specific functions. We validated 10 candidates, including the BCR signaling subunit $\alpha$ chain (Ig $/$ CD79A) and the cluster of differentiation-22 (CD22), which is an important inhibitory receptor for BCR signaling. Moreover, we identified in the intracellular domain of CD22 the tyrosine that is dephosphorylated by PTP1B. Thus, we gained new molecular insight into how PTP1B controls BCR signaling and, thereby, protects B cells from hyperactivity and auto-reactivity.

\section{Results}

\section{The human Ramos B cell proteome at absolute quantitative scale}

To explore the human B cell proteome, we performed a global quantitative MS-based proteomic study of the Burkitt's lymphoma cell line Ramos as a B cell model system. A label-free MS analysis allowed the estimation of copy numbers for 8,086 proteins by applying the proteomic ruler method (Wiśniewski et al, 2014) (Figs $1 \mathrm{~A}$ and S1A and S1B and Table S1). Copy numbers span seven orders of magnitude, varying from proteins such as the dedicator of cytokinesis protein 1 (DOCK10) with only 12 copies to the histone $\mathrm{H} 4$ with $1.13 \times 10^{8}$ copies per Ramos B cell (Fig 1A and Table S1). The large majority of proteins (80\%) exhibited copy numbers between 1,000 and 1,000,000 and 50\% between 6,500 and 165,000 copies per cell. Approximately $30 \%$ of the total protein copy number $\left(3.28 \times 10^{9}\right)$ in Ramos B cells derived from only 50 different proteins, many of which are involved in central processes including transcription, translation and energy metabolism (Fig S1C). The lymphoma character of Ramos B cells was reflected by the abundance of cancer-associated proteins including the heat shock protein 90, the cytokine macrophage migration inhibitory factor MIF, glutathione S-transferase P and peroxiredoxin-1 (Tew et al, 2011; Schulz et al, 2012; Nicolussi et al, 2017). Among the proteins with B cell-specific functions, the BCR lambda light chain variable region 2-14 exhibited the highest abundance (Table S1).

For functional data analysis, we divided the copy number dataset into five quantiles (Q1-Q5) and performed a Gene Ontology (GO) term and Reactome pathway enrichment analysis. In addition to central cellular processes such as translation and transcription, we found in each quantile proteins of distinct signaling pathways to be overrepresented (Fig 1B). Among the proteins of highest abundance (Q1), the Reactome pathway term "antigen receptor-mediated signaling pathway" was significantly enriched, highlighting the importance of BCR signaling for Ramos B cell identity, maintenance and function (Fig 1B and Table S2).

Based on our copy number data and the reported BCR signalosome network (Satpathy et al, 2015), we established an absolute quantitative map of the BCR signaling protein network (Fig 1C). The IgM class BCR (lambda light chain and $\mu$ heavy chain) and the calcium-binding protein calmodulin were most abundant followed by, for example, PLCy2, a crucial component for the initiation of $\mathrm{Ca}^{2+}$ signaling. Interestingly, the B cell co-receptors CD22, CD19, and CD81 were considerably less abundant than the IgM-BCR. Other abundant proteins were the hematopoietic lineage cell-specific protein HCLS1, which becomes tyrosine phosphorylated on multiple sites upon BCR stimulation (Yamanashi et al, 1993), and the growth factor receptor-bound protein 2 (GRB2), an central adaptor protein which couples several membrane receptors to Ras signaling (Lowenstein et al, 1992). In comparison to GRB2, the Ras guanine exchange factor son of sevenless 1 (SOS1) was found to be three orders of magnitude lower in abundance and, thus, it may represent a signaling-limiting factor in B cells. Among the identified transcription factors, interleukin enhancer-binding factor 2 (ILF2) was most abundant, pointing to an important role of this factor in modulating $B$ cell signaling responses.

\section{The abundance profiles of protein tyrosine kinases and protein tyrosine phosphatases}

Protein tyrosine phosphorylation mediated by PTKs such as the spleen tyrosine kinase (SYK), the Src family kinases (SFKs), and the Bruton tyrosine kinase (BTK), is essential for BCR activation and downstream signaling (Aoki et al, 1994; de Weers et al, 1994; Petro et al, 2000). Based on our Ramos B cell proteome data, we established the copy number profile of 28 PTKs (Fig 2A and Tables S1). The C-terminal SRC kinase CSK (617,000 copies), BTK $(363,000$ copies), and two SFKs, namely LYN isoform 1 (304,000 copies) and lymphocyte-specific protein tyrosine kinase LCK (338,000 copies) were most abundant followed by PTK2B (163,000 copies) and SYK (123,000 copies), whereas most other PTKs were considerably lower in abundance with copy numbers between 16,000 and 600 per cell. CSK negatively regulates SFKs by the phosphorylation of a carboxyterminal tyrosine and thereby limits BCR signaling (Hata et al, 1994; Chong et al, 2005). Interestingly, CSK was expressed at the same level as LYN isoform 1 and LCK together, suggesting that the expression levels of these kinases are precisely regulated.

In our study of the Ramos B cell proteome, we identified 37 PTPS of which 14 were "classical" PTPs (Figs 2B and S2A and Table S1). From the classical PTPs, the protein tyrosine phosphatase receptor type C (PTPRC, also known as CD45) and the src homology region 2 domain-containing phosphatase-1 (SHP-1) play a pivotal role in the control of BCR signaling (Cyster \& Goodnow, 1995; Cornall et al, 1998; Dustin et al, 1999; Zhu et al, 2008). Most abundant in the group of classical PTPs was CD45 (464,000 copies) with an 1.5-fold higher expression than its substrate Lyn (Katagiri et al., 1999). Interestingly, the ubiquitous phosphatase PTP1B (104,000 copies) was ranked third after SHP-2 and expressed at the same level as the kinase SYK, which is essential for BCR downstream signaling (Fig 2A and B).

A comparison of the Ramos $B$ cell proteome with the proteome of human naive $B$ cells (Rieckmann et al, 2017) revealed a high 
A

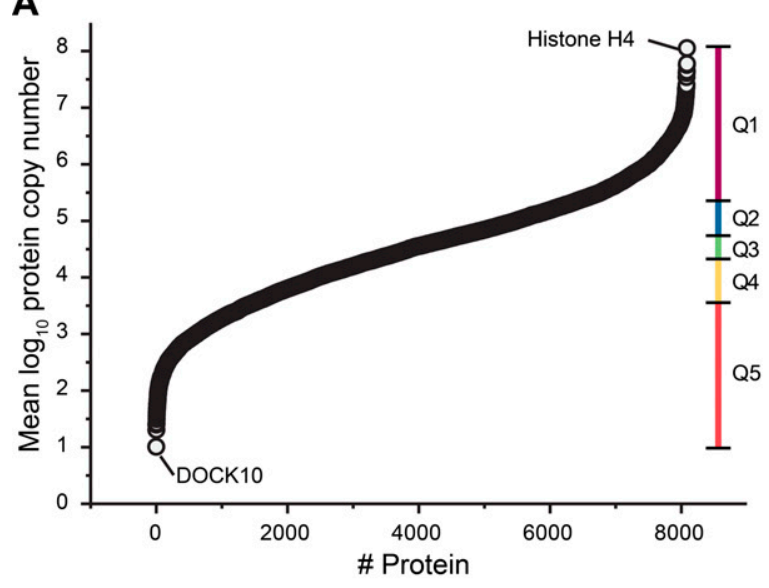

B

\begin{tabular}{|c|c|c|c|}
\hline & Term & p-value & \# Genes \\
\hline Q1 & Translation & 3.74E-94 & 251 \\
\hline QI & Antigen receptor-mediated signaling pathway & 4.19E-16 & 73 \\
\hline & rRNA processing & $1.30 \mathrm{E}-18$ & 76 \\
\hline Q2 & Interferon signaling & 1.17E-06 & 43 \\
\hline & tRNA metabolic process & 4.36E-09 & 46 \\
\hline Q3 & Toll-like receptor 9 cascade & $6.90 \mathrm{E}-03$ & 21 \\
\hline & Histone acetylation & $1.32 \mathrm{E}-10$ & 42 \\
\hline Q4 & TNF signalling & 1.85E-04 & 15 \\
\hline O5 & Microtubule organising œntre organisation & 4.71E-07 & 33 \\
\hline Q & Signaling by TGF-beta receptor œmplex & 4.21E-02 & 15 \\
\hline
\end{tabular}

C

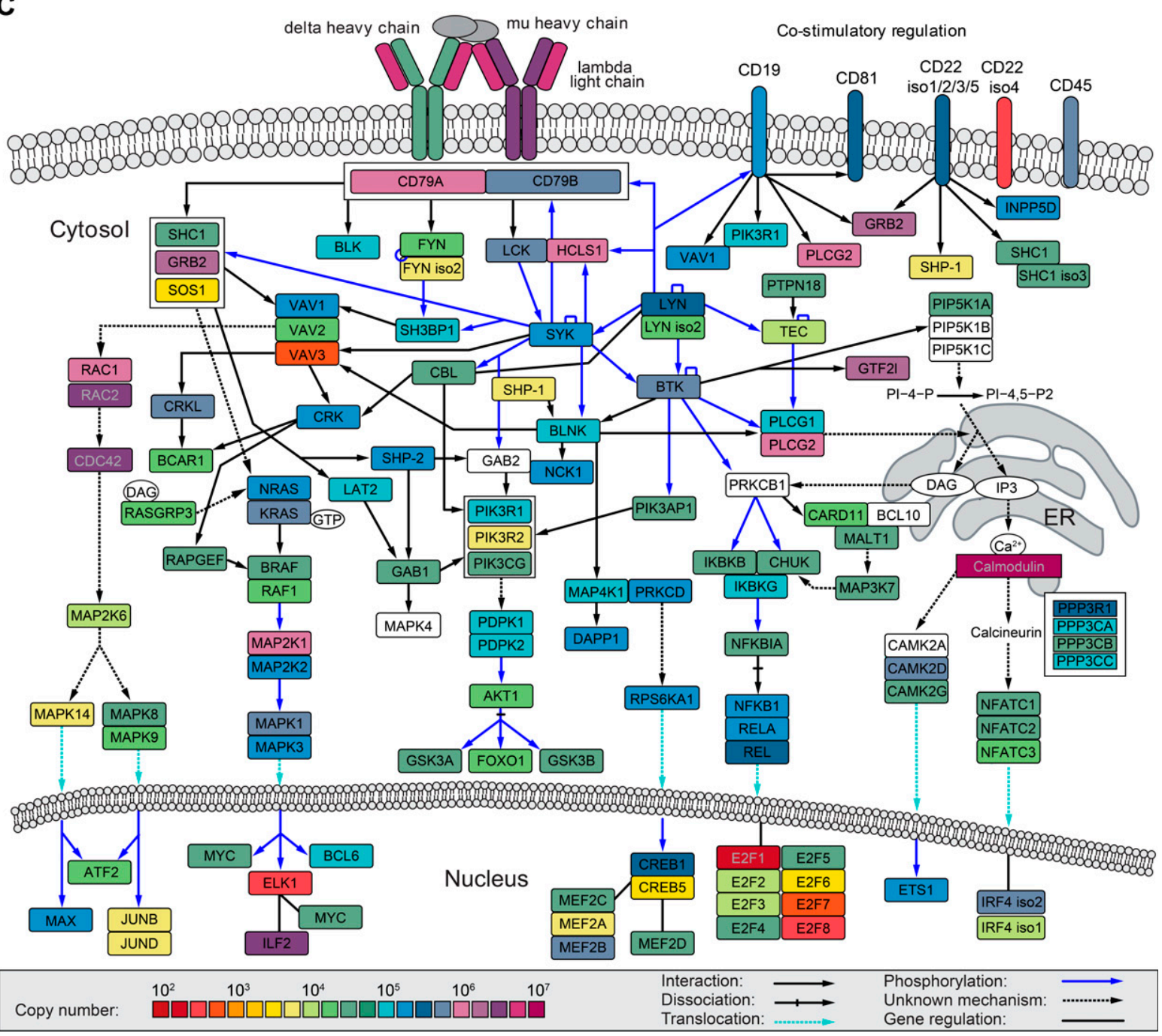

Figure 1. Global absolute quantification of protein expression in Ramos B cells.

(A) Ramos B cell protein copy number plot. Shown are the mean $\log _{10}$-transformed copy number values of 8,086 proteins. The dataset was divided into five equal-sized quantiles (Q1-Q5) according to copy number rank. (B) Gene ontology term enrichment analysis of proteins in Q1-Q5, as shown in (A), against the human proteome. For each quantile, significantly enriched processes of the Gene Ontology term "biological process" and the Reactome pathway are shown with Bonferroni step-down corrected P-values $(n=3)$ and the number of associated genes. (C) BCR downstream signaling network. The mean copy numbers of individual proteins per Ramos B cell $(n=3)$ are indicated by their color. The pathway was adapted from Satpathy et al (2015). See also Fig S1 and Table S1. 
A

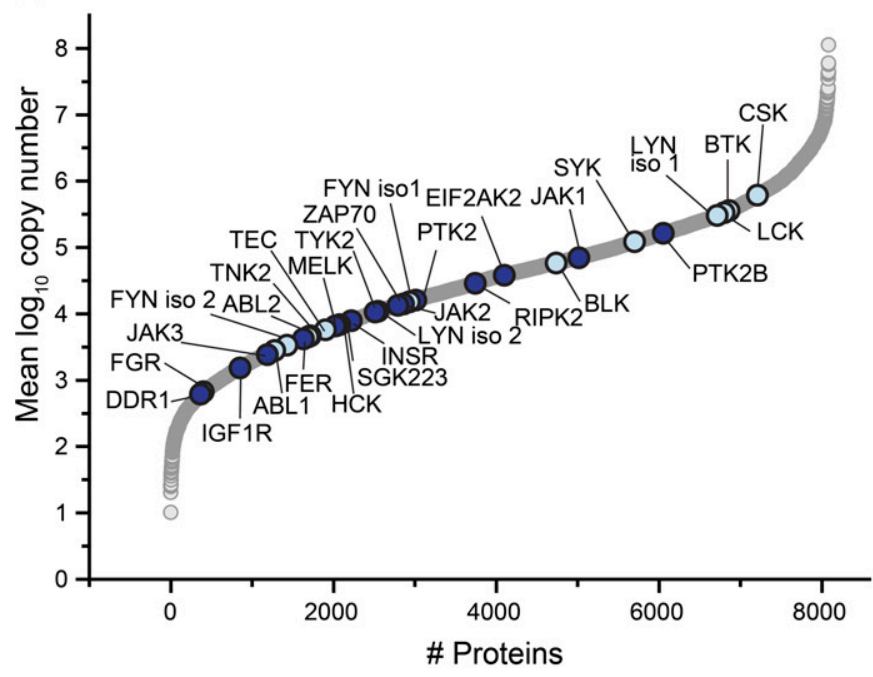

C

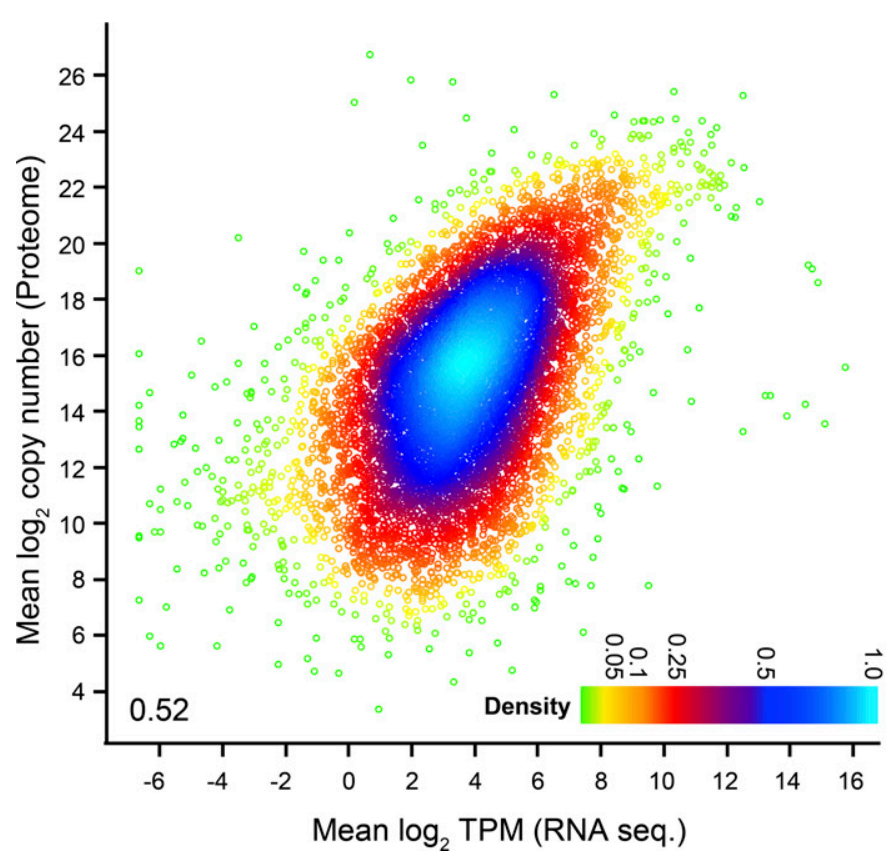

B

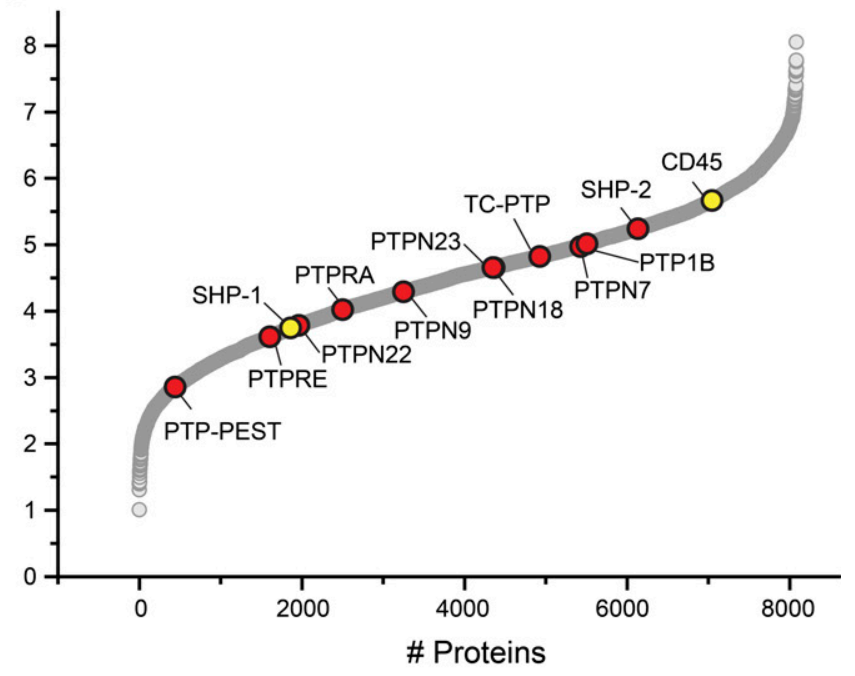

D

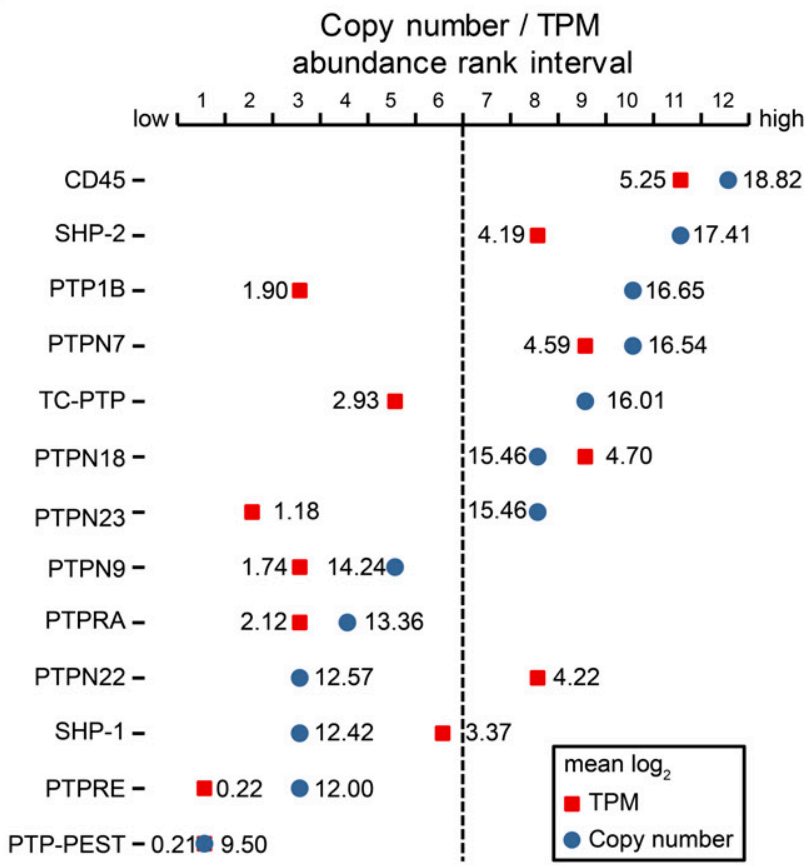

Figure 2. Evaluation of protein tyrosine kinases (PTKs) and classical protein tyrosine phosphatases (PTPs) in Ramos B cells at quantitative scale.

(A) Copy number profile of 28 different PTKs identified and quantified in Ramos B cells. Src family kinases and other PTKs known to be involved in BCR signaling are shown in light blue, all others in dark blue. (B) Copy number profile of the 13 classical PTPs identified and quantified in Ramos B cells. CD45 and SHP-1, known to be involved in BCR signaling are highlighted in yellow, the other classical PTPs in red. (C) Density plot comparing mean log-transformed protein copy numbers of the Ramos B cell proteome analysis against transcript per million values of the RNA sequencing dataset. A Pearson correlation coefficient of 0.52 was calculated. TPM, transcripts per

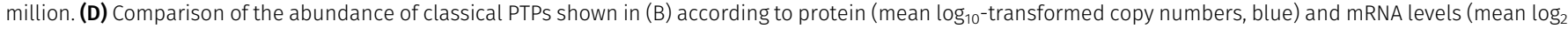
transcript per million values, red) of 7,944 proteins and transcripts, respectively. Mass spectrometric and RNA-sequencing datasets were divided into 12 equal-sized abundance rank intervals, with the least and most abundant 662 proteins/transcripts in interval 1 and 12, respectively.

correlation $(r=0.70)$ of protein abundance, including comparable levels for PTKs and classical PTPS (Fig S2B-D and Table S3). The only exception was SHP-1, which was more than 280-fold lower expressed in Ramos (5,600 copies) compared with naive B cells, whereas CD45 and PTP1B were equally abundant in the two studied $B$ cell populations (Fig S2C and D). Our findings are in line with previous work showing that SHP-1 levels are reduced to $5 \%$ in EBV-negative Burkitt lymphomas compared with normal B cells (Delibrias et al, 1997).

We next compared protein and mRNA expression levels of Ramos $B$ cells. Mean $\log _{2}$ transcript per million (TPM) values of all proteincoding transcripts (13,611 transcripts) were calculated from RNA 
sequencing data of Ramos B cells (Qian et al, 2014) (Fig S2E and Table S4). Most of the protein-coding genes followed a normal distribution with a maximum at a mean $\log _{2}$ TPM value of $\sim 4$. Low abundant transcripts caused a divergence from this distribution, but these likely represent lowly expressed genes producing nonfunctional transcripts (Hebenstreit et al, 2011). We thus excluded stochastically expressed transcripts (mean $\log _{2}$ TPM value $<0$ ). The GO term enrichment analysis confirmed that many transcripts refer to functions in other cell types (Table S5). Mapping of the functional transcripts (9,905; mean $\log _{2}$ TPM value $\geq 0$ ) to our proteome data resulted in an overlap of 7,944 proteins with a moderate correlation between TPM and copy number values $(r=0.52$ ) (Figs 2C and S2F and Table S6). We compared the expression levels of 13 classical PTPS quantified at the protein and transcript level (Tables S1 and S7). We found considerable differences between TPM and copy number values and, in most cases, the examined PTPs were more abundant at the protein level (Fig 2D). Only SHP-1, PTPN22, and PTPN18 showed higher transcript levels, which points to posttranscriptional or posttranslational mechanisms that negatively regulate their protein levels. Notably, PTP1B showed the largest discrepancy between transcript and protein levels in B cells. Because of its high abundance at the protein level, a role of PTP1B in B cell signaling can be anticipated.

\section{Loss of PTP1B leads to altered protein phosphorylation upon BCR stimulation}

To investigate the function of PTP1B in Ramos B cells, we generated PTP1B KO cells using the CRISPR-Cas9 technology (Cong et al, 2013). The PTP1B deficiency was confirmed by immunoblot and MS analysis (Fig S3A and B) and quantitative proteomic analysis showed that loss of PTP1B did not result in global changes in the B cell proteome (Fig S3C and Table S8).

To reveal the impact of PTP1B on B cell signaling, changes in tyrosine phosphorylation levels of downstream signaling factors following BCR activation with anti- $\lambda$ light chain antibody were monitored for different time points (Fig 3A). After 3 and 10 min of BCR stimulation, phosphorylation of SYK at Y525/526 was significantly increased in PTP1B KO compared with WT Ramos B cells (Fig $3 A$ and B). In addition, ERK-pT202/Y204 levels were significantly increased in PTP1B KO cells (Fig 3A and C).

To show that changes in phosphorylation depend on PTP1B, we reintroduced FLAG-PTP1B in the KO cells using a two component tetracycline-inducible expression system (Haug et al, 2015). After BCR stimulation with anti- $\lambda$, the SYK-pY525/526 levels were decreased in the FLAG-PTP1B expressing PTP1B KO cells (Fig 3D and E). Similarly, ERK-pT202/Y204, BTK-pY223, and PLCy2-pY759 levels were increased in PTP1B KO cells and decreased upon FLAG-PTP1B expression (Fig 3D and 3F-H).

Taken together, our data point to a regulatory role of PTP1B in $\mathrm{BCR}$ signaling by limiting the activation of the downstream signaling components.

To analyze the role of PTP1B in intracellular calcium signaling, we measured calcium mobilization after anti- $\lambda$ stimulation of the BCR by flow cytometry in PTP1B KO cells with inducible expression of GFP (control) or FLAG-PTP1B (Fig S4A and B). In Ramos B cells expressing FLAG-PTP1B as compared with PTP1B KO cells, a similar strong influx of $\mathrm{Ca}^{2+}$ ions from the extracellular space into the cytosol was observed. However, the $\mathrm{Ca}^{2+}$ levels return faster to the baseline (i.e., level before anti- $\lambda$ stimulation) in PTP1B KO cells as compared with FLAG-PTP1B-expressing cells (Fig S4B), which is most apparent at the $300 \mathrm{~s}$ time point (Fig S4C). This finding indicates a role of PTP1B in restoring the steep calcium concentration gradient between the cytosol and the plasma membrane after a signaling event.

\section{Global analysis of the PTP1B-associated protein network in Ramos B cells}

To obtain insight into the molecular mode of action by which PTP1B restricts BCR signaling, we generated a FLAG-tagged PTP1B D181A-Y46F substrate trapping mutant (Boubekeur et al, 2011), which stabilizes the enzyme-substrate complex and thus allows the recovery of bound tyrosine-phosphorylated substrates by co-immunoprecipitation. In addition, we generated a truncated version that lacks the carboxy-terminal ER-targeting domain ( $\triangle 406$ 435) and localizes to the cytosol (Fig S5A-C) to address the question whether the substrate spectrum of PTP1B is determined by its subcellular localization. With this aim, we performed a quantitative substrate trapping study that enables one to identify and directly compare substrates and/or binding partners of full-length and truncated PTP1B via stable isotope labelling by amino acids in cell culture (SILAC)-MS (Fig 4A). Proteins with a mean SILAC ratio $\geq 2$ and a $P$-value $<0.05(n=3)$ were considered as potential PTP1B substrates and/or binding partners (Fig 4B and C and Table S9). Interestingly, the 12 proteins bound to the truncated cytosolic form of PTP1B represent only a subset of the 22 candidates identified for full-length PTP1B that harbors the ER-targeting domain (Fig 4D). These PTP1B interactors include the adaptor protein GRB2, PLCY1, and Rho GTPase-activating protein 12 (ARHGAP12), which have been earlier identified as PTP1B substrates/interactors in other species or cell types (Liu et al, 1996; Mertins et al, 2008; Ferrari et al, 2011; Banh et al, 2016). More than one-third of the identified substrate candidates carry a SH3 domain (Table S9), suggesting that they bind to the proline-rich region of PTP1B. In comparison, only 2\% of all proteins in the human proteome carry a SH3 domain according to the SMART database (Letunic et al, 2015). Network analysis using the STRING database further allowed a classification of the candidates into three main functional groups associated with the GO terms "meiotic cell cycle regulation," "cargo recognition for clathrinmediated endocytosis (CME)," and "BCR signaling" to which 10 proteins were assigned (Fig 4E and Table S9). These data indicate that ER-anchored PTP1B specifically targets a set of proteins with key functions in B cell signaling including the kinase BTK, the inhibitory co-receptor CD22, and the BCR subunits CD79A (Ig $\alpha$ ) and CD79B $(\lg \beta)$. Several of the identified PTP1B interactors are involved in calcium signaling and we therefore investigated whether or not the expression of the trapping mutant affects calcium mobilization in comparison to PTP1B KO cells with inducible expression of GFP as a control (Fig S6A and B). Indeed, the expression of FLAG-PTP1B D181A-Y46F in PTP1B KO cells strongly reduces the overall calcium flux (Fig S6B and D), indicating that the trapping mutant is capturing a significant pool of substrate candidates that play a role in calcium signaling. Expression of FLAG-PTP1B D181A-Y46F $\triangle 406-$ 435 that lacks the ER-targeting domain also interferes with calcium 
A

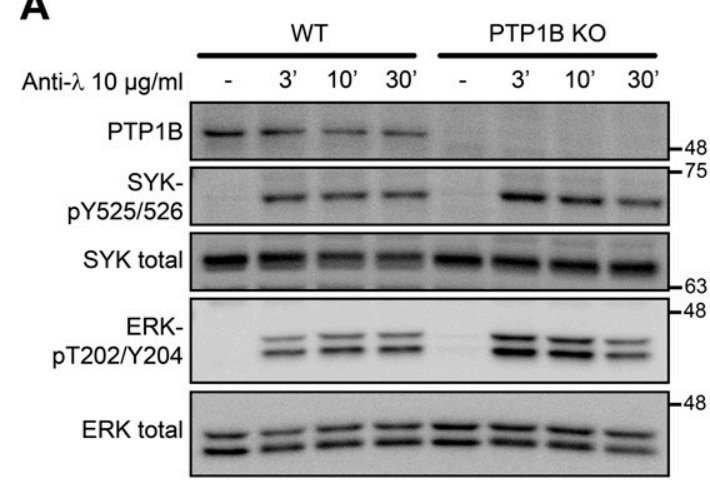

D

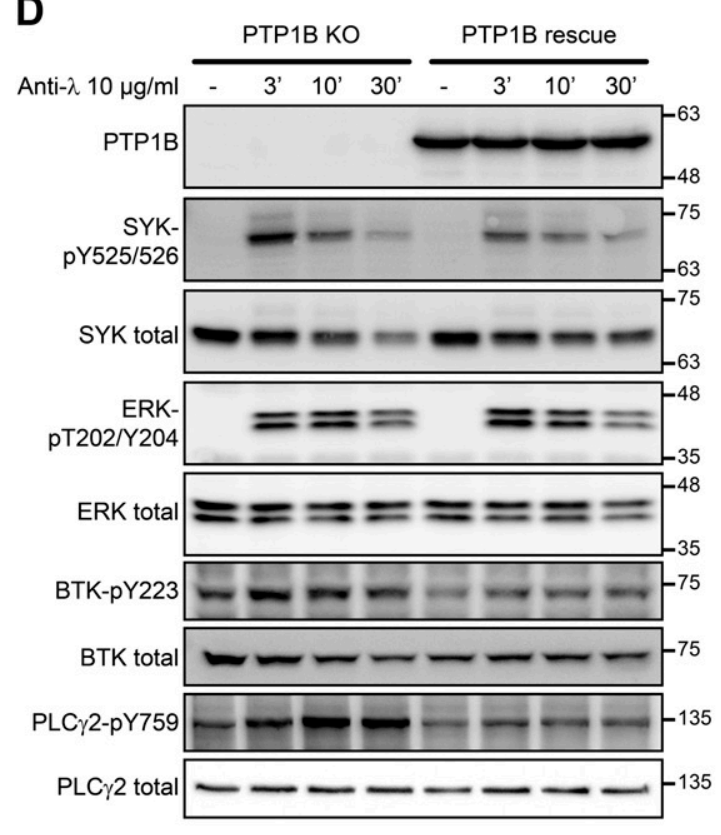

B

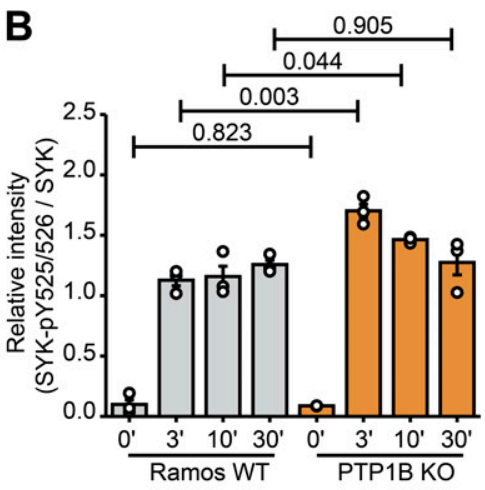

E
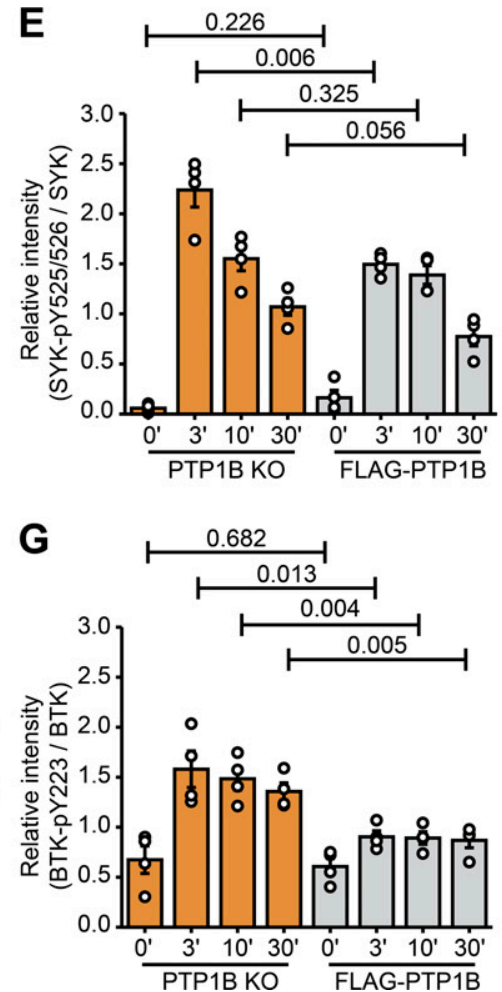

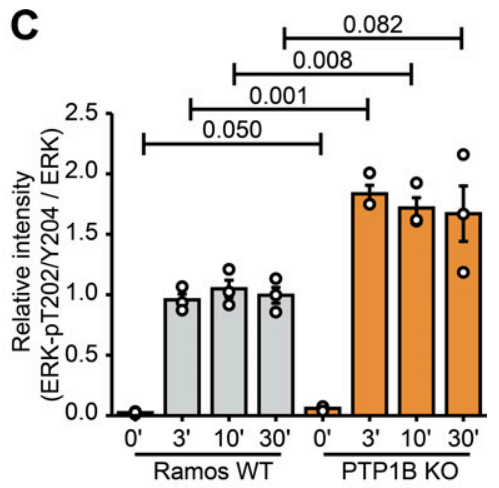

$\mathbf{F}$
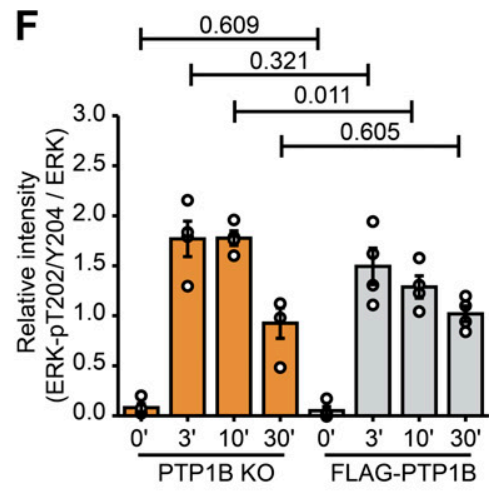

H
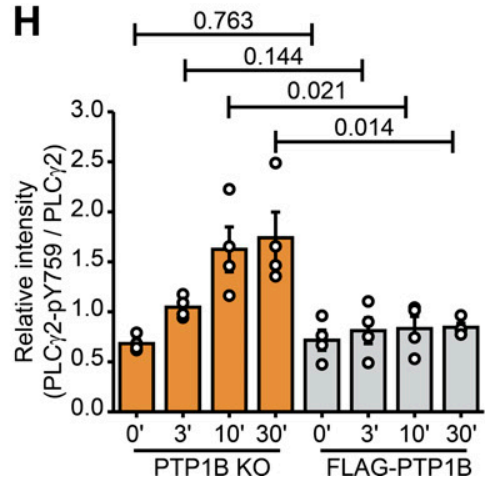

Figure 3. Loss of PTP1B leads to an increase in phosphorylation of SYK-Y525/526, ERK-T202/Y204, PLCY2-Y759, and BTK-Y223 in Ramos B cells.

(A) Immunoblot analysis of SYK-Y525/526 and ERK-T202/Y204 phosphorylation in WT and PTP1B KO Ramos cells stimulated with anti- $\lambda$ for the indicated time points. Representative immunoblots of three independent biological replicates are shown. (B, C) Quantification of immunoblot data of SYK-pY525/526 (B) and ERK-pT202/Y204 (C) shown in (A). Signal intensities were normalized to the respective total protein signals and a $t$ test was performed $(n=3)$. Error bars represent the SEM. (D) Immunoblot analysis of PTP1B KO and FLAG-PTP1B induced (PTP1B rescue) Ramos cells stimulated with anti- $\lambda$ for the indicated time points. Representative immunoblots of three independent biological replicates are shown. (E, F, G, H) Quantification of immunoblot data of SYK-pY525/526 (E), ERK-pT202/Y204 (F), BTK-pY223 (G), and PLCY2-pY759 (H) shown in (D). Signal intensities were normalized to the respective total protein signals and a $t$ test was performed $(n=4)$. Error bars represent the SEM. Source data are available online for this figure.

signaling, but has a milder effect as compared with the full-length trapping mutant (Fig S6C and D).

\section{Validation of B cell-specific PTP1B substrates}

For validation of our PTP1B substrate network data, we selected 10 proteins with a focus on proteins involved in BCR signaling and performed anti-FLAG co-immunoprecipitations using PTP1B KO Ramos B cells expressing a FLAG-tagged version of either PTP1B WT or the full-length and truncated form of PTP1B D181A-Y46F (Fig 5A). The immunoblot data show that Ig $\alpha$ and CD22 exclusively interact with the full-length trapping mutant, which indicates that PTP1B's localization at the ER is essential for this interaction. In contrast, PLCY1 and PLCY2 as well as the B cell adapter for phosphoinositide 3-kinase (BCAP), the amyloid-like protein 2 (APLP2), and the E3 ubiquitin-protein ligase CBL were recovered with both PTP1B trapping mutant versions, with even higher binding to the cytosolic form of PTP1B. Effective binding to the full-length PTP1B trapping 
A

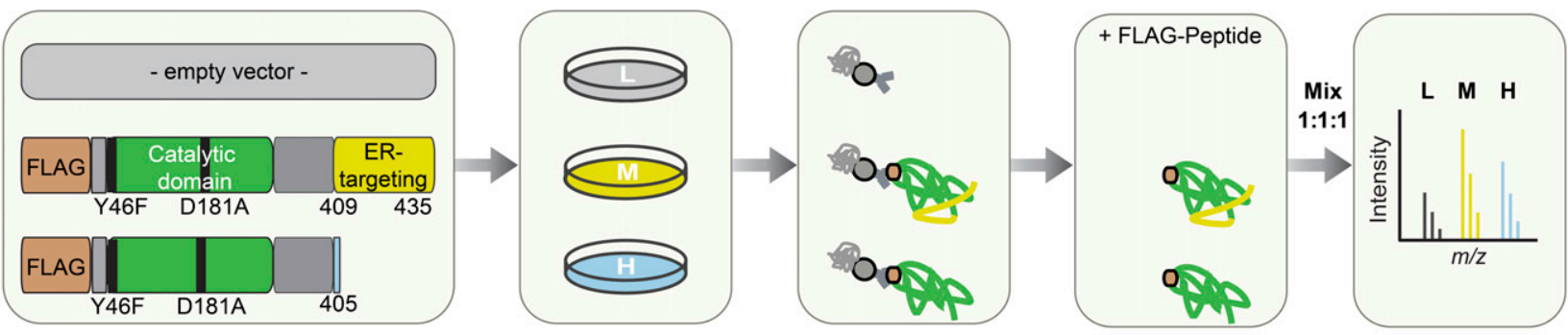

B

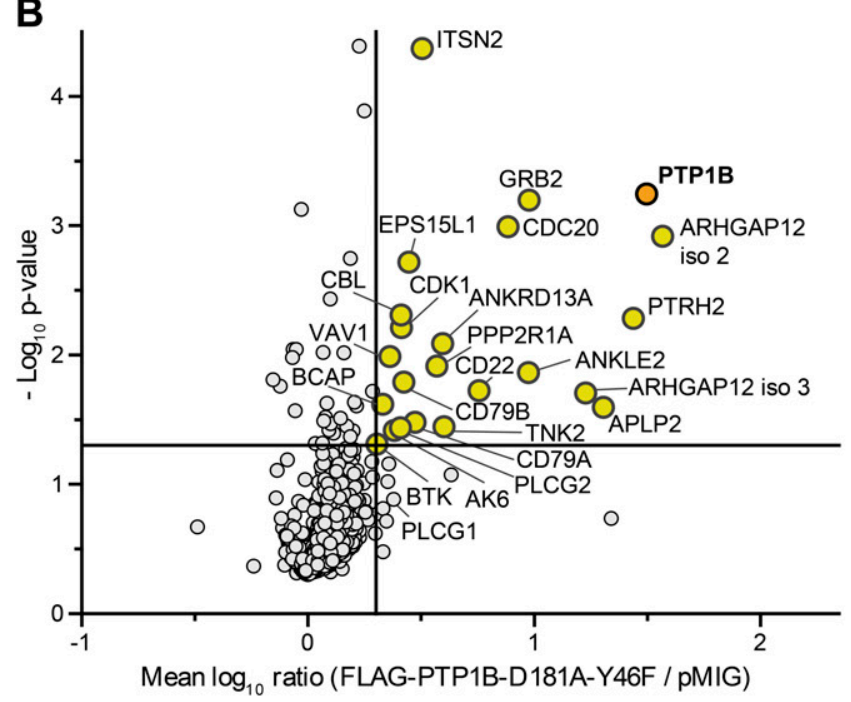

C

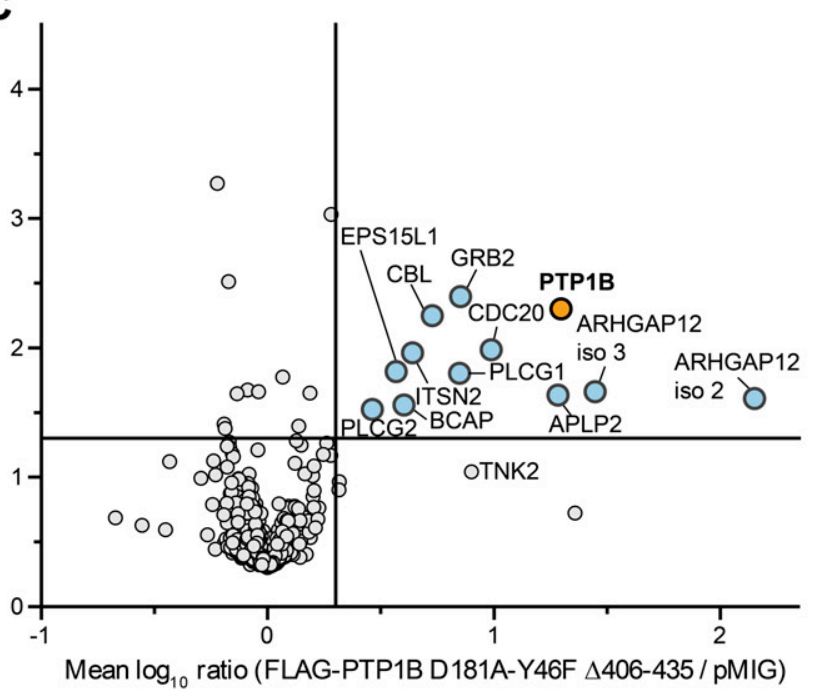

D

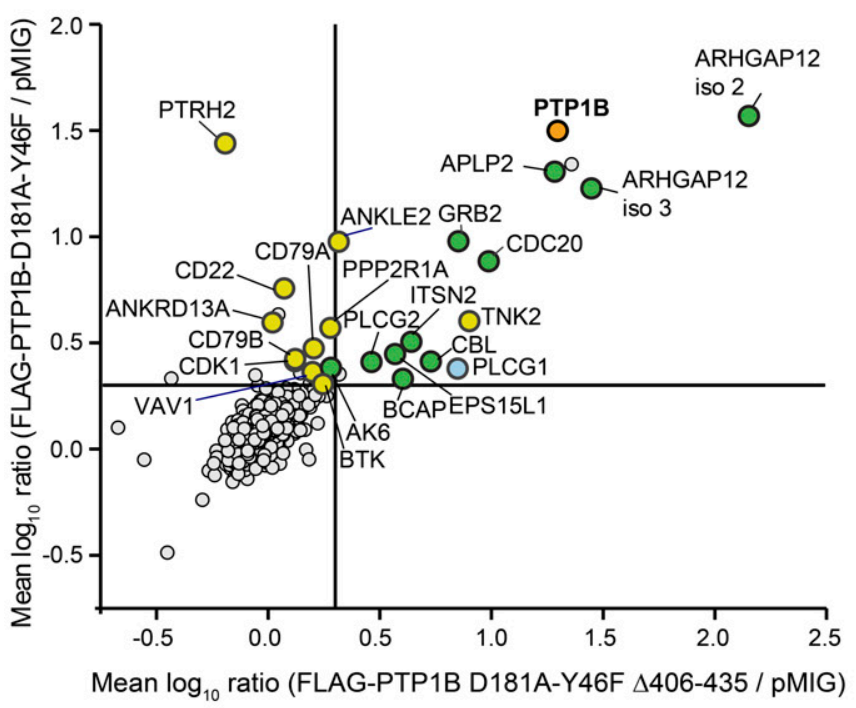

E

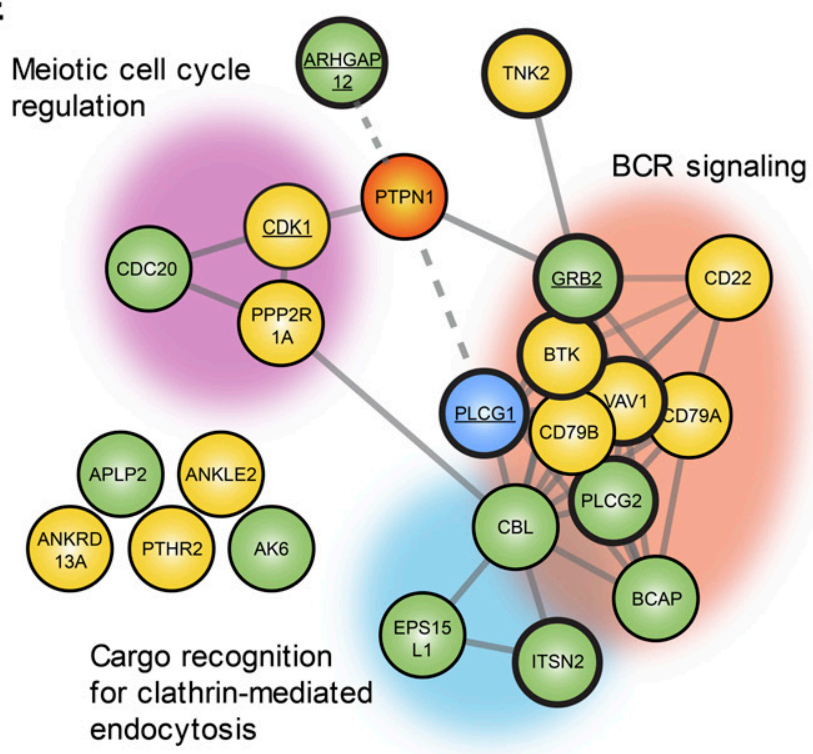

Figure 4. Identification of putative PTP1B substrates in Ramos B cells.

(A) Experimental design. Ramos PTP1B KO cells stably expressing the empty vector control, the FLAG-tagged PTP1B-D181A-Y46F trapping mutant or the FLAG-tagged truncated trapping mutant without the ER-targeting domain were subjected to stable isotope labelling by amino acids in cell culture (SILAC) using "light" (L), "mediumheavy" (M), and "heavy" $(\mathrm{H})$ versions of arginine and lysine. Following SILAC, co-immunoprecipitations using anti-FLAG beads were performed. Bound proteins were eluted by competition with FLAG peptide and eluates were mixed in equal ratio. SILAC samples were separated by SDS-PAGE followed by in-gel digestion of proteins using trypsin and quantitative LC-MS analysis. (B, C) Scatterplot of proteins eluted with the trapping mutant FLAG-PTP1B-D181A-Y46F (B) and the truncated form of FLAG-PTP1BD181A-Y46F lacking amino acid residues $\triangle 406-435$ (C). Mean $\log _{10}$-transformed SILAC ratios were plotted against - $\log _{10} P$-values. Proteins with a minimum fold change of two and a $P$-value $<0.05$ ( $n=3$; right-sided $t$ test) are highlighted in yellow (B) or in blue (C). The bait PTP1B is shown in orange. (D) Scatterplot of proteins eluted with 
mutant was found for BTK, APLP2, and the adaptor proteins GRB2 and guanine nucleotide exchange factor VAV1 all of which also interacted with PTP1B WT to different extent (Fig 5A).

To further distinguish between PTP1B-binding partners and bound substrates, we used orthovanadate to release the substrate from the catalytic centre of PTP1B by binding competition. In the presence of orthovanadate, the interaction with Ig $\alpha, \mathrm{CD} 22, \mathrm{PLCY} 1 / 2$, $\mathrm{CBL}$, and BCAP was virtually abolished, which identifies them as PTP1B substrates (Fig 5B). However, the binding of BTK, VAV1, and GRB2 was only reduced, suggesting that this binding is mediated by the $\mathrm{SH} 3$ domain of these proteins interacting with the proline-rich sequences of PTP1B.

The interaction of PTP1B with its substrates Ig $\alpha, C D 22$, and APLP2 was confirmed by an in situ proximity ligation assay (PLA). We observed signals (dots per cell) for PTP1B/Ig $\alpha$, PTP1B/CD22, and PTP1B/APLP2 that were significantly higher in WT than PTP1B KO cells (Fig 5C), underscoring the specific interaction of these proteins with PTP1B in Ramos B cells.

In summary, we validated Ig $\alpha, C D 22, P L C y 1 / 2, C B L, B C A P$, and APLP2 as in vivo substrates of PTP1B in B cells. In addition, BTK and the adaptor proteins GRB2 and VAV1 were identified as direct binding partners and potential dephosphorylation targets of PTP1B.

\section{PTP1B dephosphorylates specific tyrosine residues in CD22}

Among the validated PTP1B substrates was the inhibitory receptor CD22 that counteracts BCR signaling. CD22 carries six tyrosine residues within its intracellular tail sequence, of which three are located within the immunoreceptor tyrosine-based inhibition motifs (ITIMS) of CD22 and one in the ITIM-like motif (Fig 6A). All six tyrosine residues are conserved between mouse and human CD22 and with the exception of $Y 767 / Y 752$, all of them are known to be phosphorylated in vivo (Schulte et al, 1992; Cornall et al, 1998; Yohannan et al, 1999). After BCR stimulation, GRB2 and SHP-1 are recruited to CD22 (Law et al, 1996; Otipoby et al, 2001). To identify the tyrosine residue(s) in CD22 that are dephosphorylated by PTP1B, we carried out a peptide-based phosphatase assay. We were able to synthesize four different peptides of human CD22 carrying a central phospho-tyrosine ( $\mathrm{pY}$ ) and sequences from the first ITIM (Y762), the ITIM-like (Y796), the second ITIM (Y822), and the GRB2-binding region (Y807). The other two peptides carrying pY752 and pY842 were synthetically not accessible. With these four peptides, we measured the velocity of the dephosphorylation reaction with increasing concentrations of the respective pY-peptides (Fig 6B). In comparison to the control peptide KKKKPYPKK (Li \& Köhn, 2016), all four CD22-pY-peptides were dephosphorylated by recombinant PTP1B. Based on these data, we conclude that these CD22-pY residues are substrates of PTP1B in vitro.

To examine PTP1B target sites in CD22 in a cellular context, we individually exchanged each of the tyrosine to phenylalanine to prevent phosphorylation. In addition, CD22 mutants were generated in which the tyrosine residues in the second (Y822) and third ITIM (Y842), all ITIMs (Y762, Y822, and Y842) or all six tyrosines (6Y-to-F mutant) in its intracellular part were replaced by phenylalanine. Carboxy-terminally Myc-tagged versions of CD22 WT and the different non-phosphorylatable CD22 mutants were stably expressed in PTP1B KO Ramos B cells with inducible expression of the FLAGPTP1B D181A-Y46F trapping mutant. Because substrate binding is much stronger upon tyrosine phosphorylation, we assumed that tyrosine-to-phenylalanine mutations should reveal the CD22 site(s) targeted by PTP1B. Immunoblot analysis showed that the PTP1B trapping mutant was effectively co-purified with CD22-Myc in Ramos B cells, whereas this interaction was abolished for the $6 \mathrm{Y}$-to$F$ mutant of CD22 (Fig 6C and D). These data confirm that CD22 tyrosine phosphorylation is required for PTP1B binding. A mutation of all three ITIM tyrosine residues to phenylalanine (Y762/822/842F) leads to a strong decrease in overall CD22 tyrosine phosphorylation, whereas the $\mathrm{Y} 822 / \mathrm{Y} 842 \mathrm{~F}$ double mutation has only little impact on the tyrosine phosphorylation status of CD22 (Müller et al, 2013; Chen et al, 2016). In line with these findings, the interaction of the PTP1B trapping mutant with the CD22 triple mutant was significantly reduced, which was not the case for the CD22-Y822/842F double mutant. PTP1B binding was also not affected by single $Y$-to- $F$ mutations in the ITIMS of CD22, which indicates that Y762, Y822, and Y842 are not preferred target sites of PTP1B in Ramos B cells.

In our assay, we found a strongly impaired PTP1B binding for the CD22-Y807F mutant (Fig 6D). Our data therefore point to CD22-pY807 as the preferred target site of PTP1B. Of note, human CD22-Y807 (see Fig $6 \mathrm{~A})$, is part of the $\mathrm{Y}-\mathrm{E} / \mathrm{Q}-\mathrm{N}-\Psi$ motif ( $\Psi$, hydrophobic amino acid) that when phosphorylated serves as the binding site for the $\mathrm{SH} 2$ domain of GRB2 (Poe et al, 2000; Otipoby et al, 2001; Kessels et al, 2002; Chen et al, 2016). Accordingly, GRB2 binding was reduced to background levels in immunoprecipitates of the CD22-Myc Y807F mutant in Ramos B cells (Fig 6C).

To further validate CD22-pY807 as a PTP1B substrate, we performed a cellular PTP1B phosphatase assay using our FLAGPTP1B-inducible Ramos cells (Figs 6E and S7). After anti- $\lambda$ stimulation of the BCR, CD22 is phosphorylated at Y807 and the phosphorylation level is significantly reduced in cells expressing FLAG-PTP1B (Fig 6F).

Taken together, we showed that PTP1B dephosphorylates CD22pY762, -pY796, -pY807, and -pY822 in vitro and identified pY807 as the main target site of PTP1B in Ramos B cells.

\section{Discussion}

Mice with a B cell-specific PTP1B gene knockout develop systemic autoimmunity upon ageing, indicating an important regulatory role for PTP1B in B cell activation (Medgyesi et al, 2014). In this work, we

both trapping mutants. Mean $\log _{10}$-transformed SILAC ratios of proteins in (B, C) were plotted against each other. Proteins considered significantly enriched (minimum fold change of two and a P-value < 0.05) with both trapping mutants are marked in green, with FLAG-PTP1B-D181A-Y46F or FLAG-PTP1B-D181A-Y46F $\triangle 406-435$ only in yellow and blue, respectively. (E) STRING database analysis of proteins enriched with the PTP1B substrate trapping mutants. Candidates are grouped into three clusters according to the indicated Gene Ontology terms. src-homology 3-domain containing proteins are highlighted by bold margins. Known PTP1B (gene name PTPN1) interactors/substrates are underlined. 
A

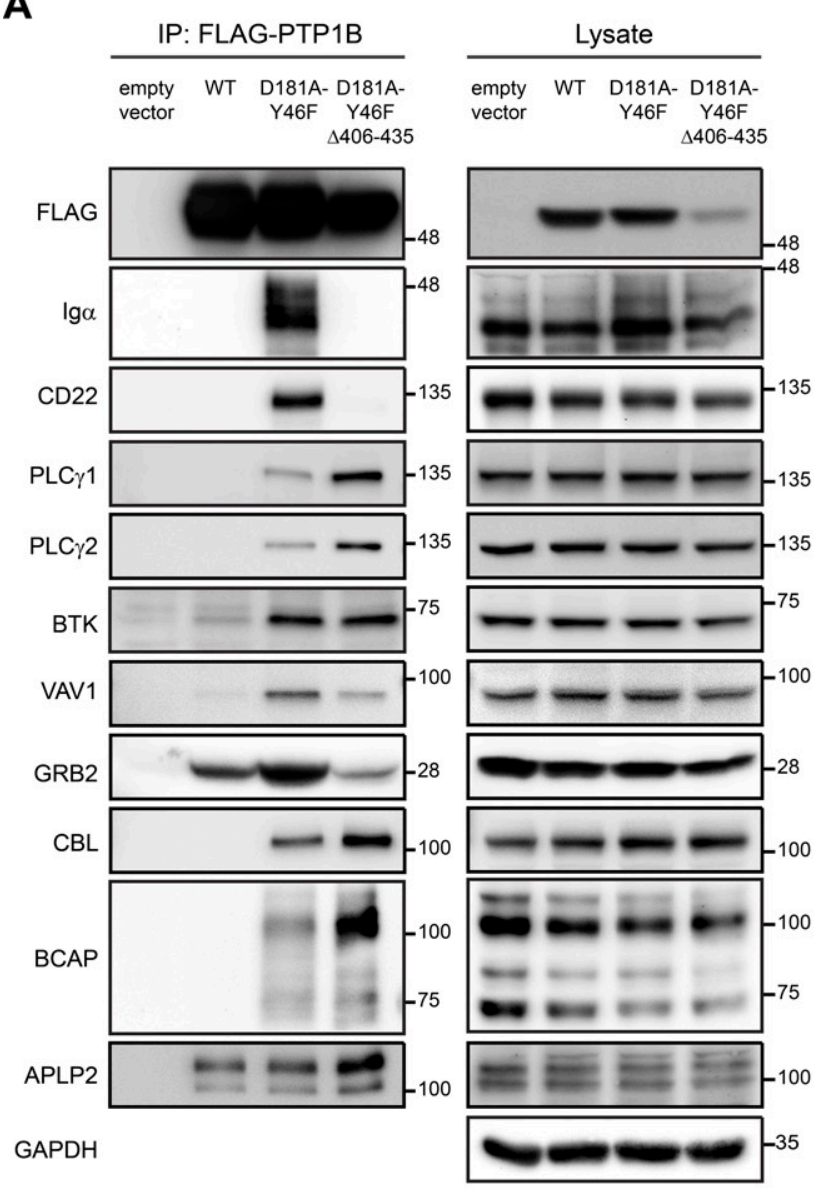

B

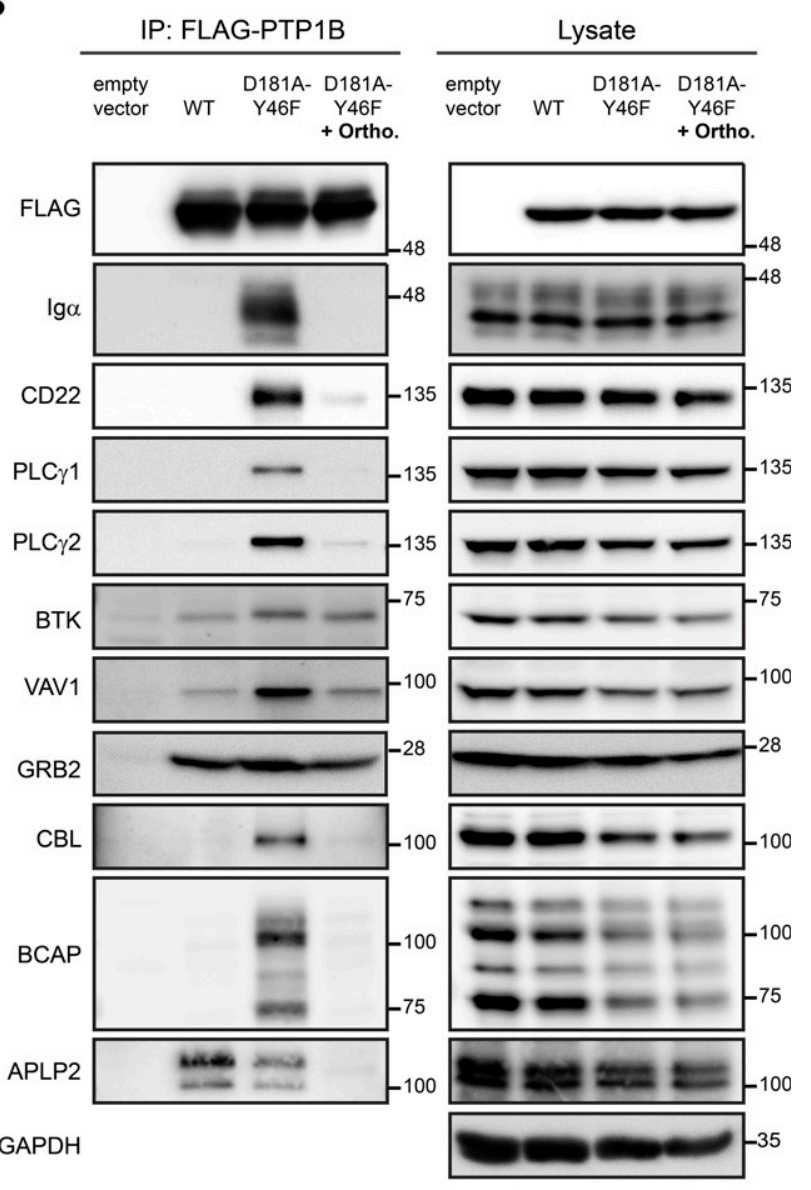

C
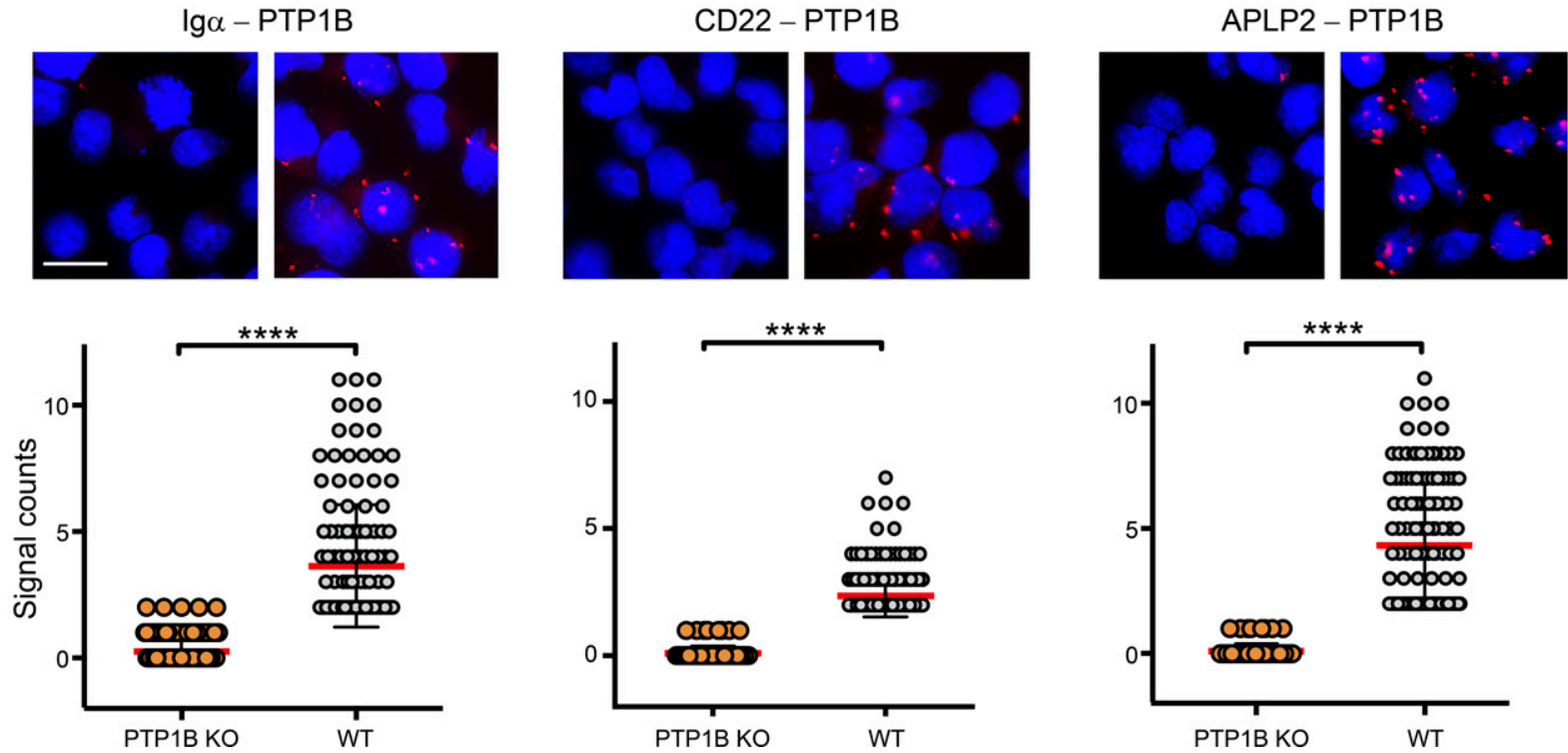

Figure 5. Validation of PTP1B substrates in Ramos B cells.

(A, B) Co-immunoprecipitation experiments were performed using PTP1B KO cells with inducible expression of empty vector control, FLAG-PTP1B WT, FLAG-PTP1BD181A-Y46F, or FLAG-PTP1B-D181A-Y46F 4 406-435 substrate trapping mutants. Eluates were subjected to SDS-PAGE and selected PTP1B substrate candidates (see Fig 4) were analyzed by immunoblotting (A). To further confirm substrates of PTP1B, cells were lysed in the presence of 1 mM orthovanadate competing for binding and, thus, promoting the release of bound substrate (B). IP, immunoprecipitation; ortho, orthovanadate. (C) PTP1B KO and WT Ramos B cells were analyzed for the interaction of PTP1B with endogenous Ig $\alpha$, CD22 or APLP2 by proximity ligation assay. Data analysis was performed with the CellProfiler software. Each dot represents one cell. Statistical analysis was performed using a Mann-Whitney test (**** $\mathrm{P}<0.0001, \mathrm{n}=3$ ). Scale bar $10 \mu \mathrm{m}$.

Source data are available online for this figure. 
A

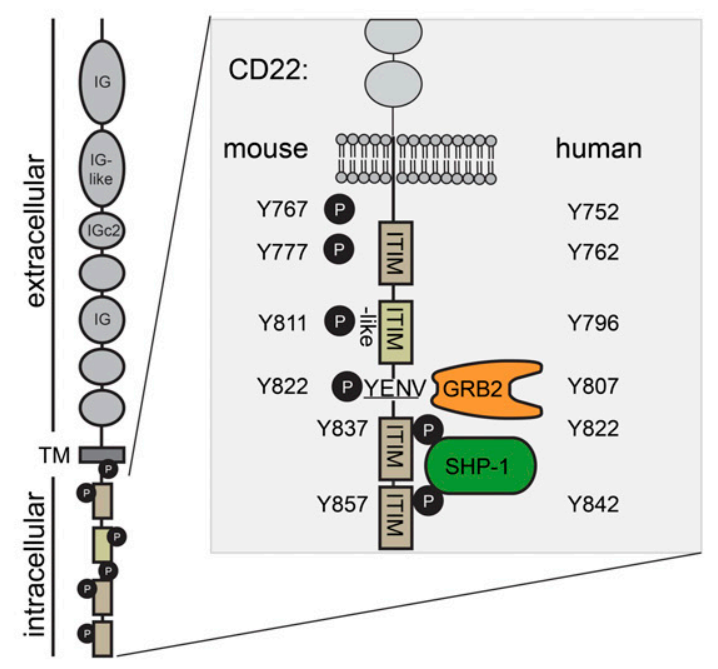

C
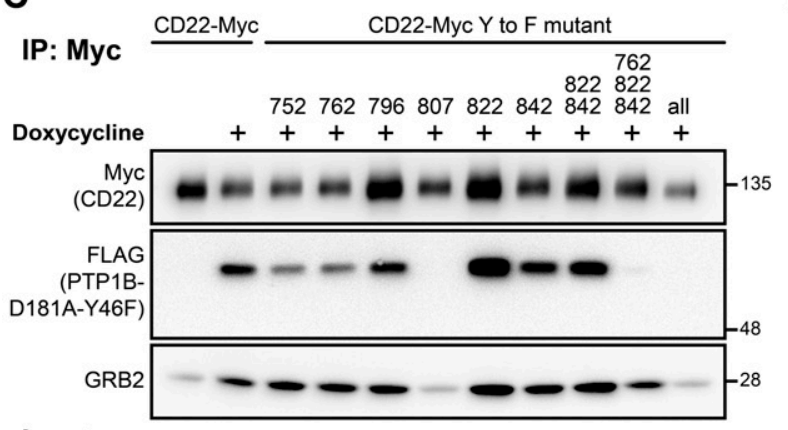

Input

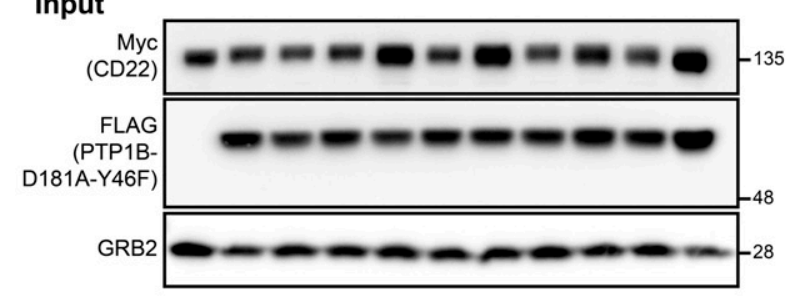

B

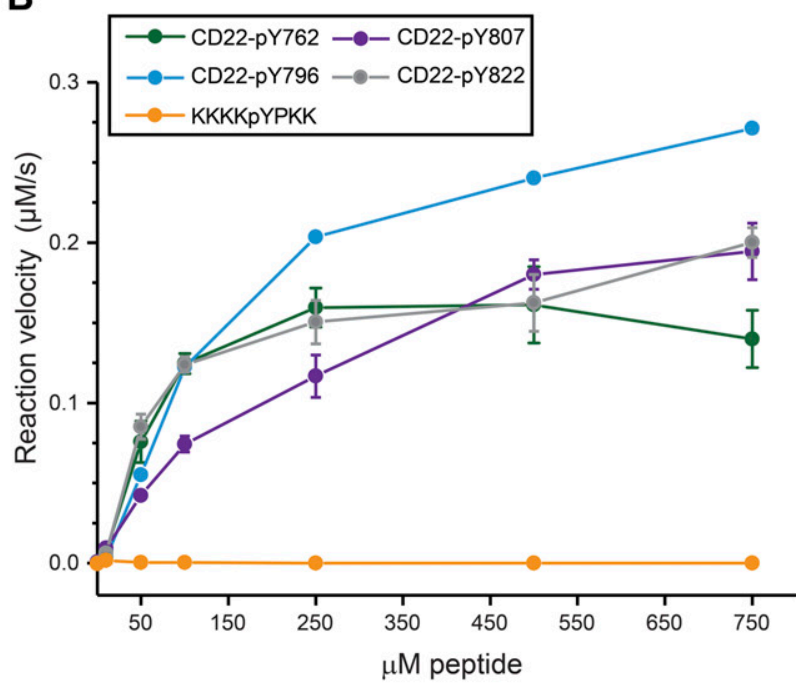

D

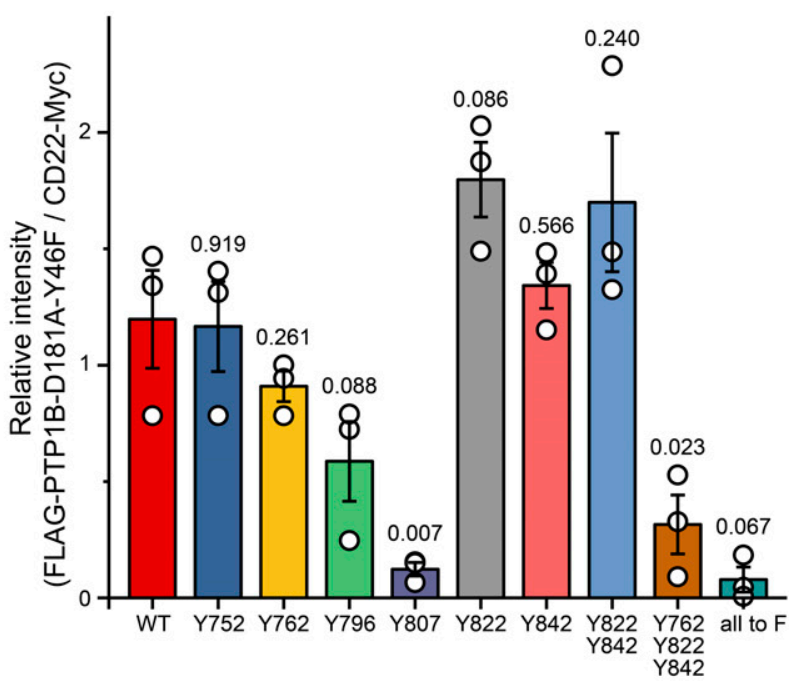

E

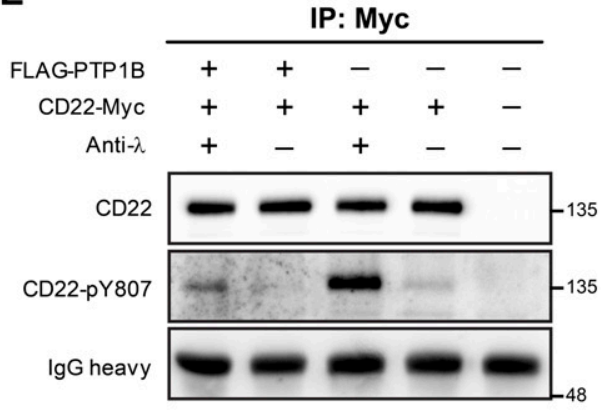

$\mathbf{F}$

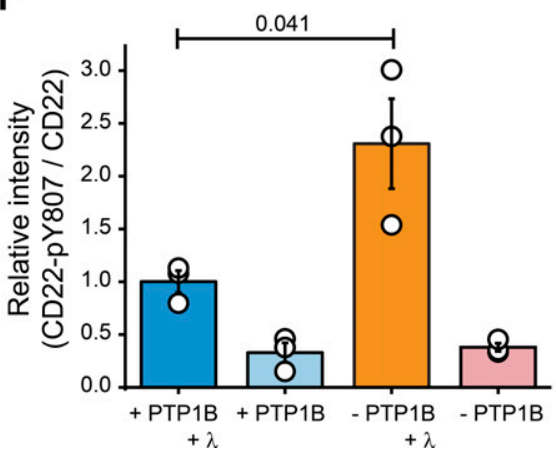

Figure 6. PTP1B dephosphorylates CD22 at pY807.

(A) Schematic representation of the human/mouse CD22 molecule with domain structure and intracellular tyrosine (Y) residues. ITIM, immunoreceptor tyrosine-based inhibitory motif; TM, transmembrane domain; IG, immunoglobulin domain; GRB2, growth factor receptor-bound protein 2; SYK, spleen tyrosine kinase. (B) Peptide-based phosphatase assay showing the dephosphorylation of four synthetic CD22 peptides by GST-tagged PTP1B. Error bars represent the standard deviation of three independent experiments. (C) PTP1B KO Ramos cells with inducible FLAG-PTP1B-D181A-Y46F trapping mutant were stably transduced with Myc-tagged CD22 WT or Myctagged CD22 site mutants in which single tyrosine residues were replaced by phenylalanine ( $\mathrm{Y}$-to-F mutants) as indicated. Following inducible expression of FLAG-PTP1BD181A-Y46F, co-immunoprecipitations using anti-Myc antibody were performed and bound proteins were eluted $(n=3)$. CD22 WT and phosphosite mutants were detected 
aimed at a better understanding of the molecular mode of action of PTPB1 in human B cells using quantitative proteomic and biochemical methods. For our systematic analysis, we chose the Burkitt lymphoma cell line Ramos as a model for mature human B cells. Our absolute proteome-wide quantification revealed a high abundance of PTP1B in Ramos B cells consistent with reported data from naive B cells (Rieckmann et al, 2017). The necessity of quantification at the level of proteins was highlighted by comparing protein copy numbers determined in this work and TPM values calculated from RNA sequencing data of human Ramos B cells (Qian et al, 2014). This revealed a considerably higher expression of PTP1B on the protein than on the transcript level. We also compared the abundance of other PTPS regulating B cell signaling such as SHP-1 and CD45 (Cornall et al., 1998; Cyster and Goodnow, 1995; Dustin et al., 1999; Katagiri et al., 1999; Pani et al., 1995; Zhu et al., 2008). We found that SHP-1 was significantly more abundant in naive B cells than in Ramos $B$ cells, whereas CD45 copy numbers were consistently high in both of these cells.

Loss of PTP1B in Ramos B cells did not affect steady-state protein levels as shown by SILAC-MS analysis. However, loss of PTP1B altered $B C R$ downstream signaling shown by a significant increase in the levels of SYK-pY525/526, ERK-pT202/Y204, BTKpY223, and PLCY2-pY759. These data point to a role of PTP1B in counteracting BCR signaling and, thus, B cell activation. PTP1B was previously shown to dephosphorylate SYK at pY526 in isolated chronic lymphocytic leukemia cells from patients (Boelens et al, 2009).

In a quantitative substrate trapping approach, we identified 22 potential targets of PTP1B in human Ramos B cells with functions in meiotic cell cycle regulation as well as cargo recognition for clathrin-mediated endocytosis and BCR signaling, two functionally interconnected processes. PTP1B has previously been implicated in the regulation of the cell cycle because it is a target of CDK1 in mitotic cells (O'Donovan et al, 2013). The three PTP1B substrate candidates CBL, intersectin-2 (ITSN2), and epidermal growth factor receptor substrate 15-like 1 (EPS15L1) are associated with clathrincoated pits and were shown to be tyrosine phosphorylated upon antigen stimulation of the BCR (Matsumoto et al, 2009; Satpathy et al, 2015). In addition, the ubiquitin-binding protein ankyrin repeat domain 13a (ANKRD13A), a protein involved in EGFR endocytosis, was found in BCR signalosomes (Tanno et al, 2012; Satpathy et al, 2015). In fact, it was reported that PTP1B regulates the endosomal trafficking of RTKs (Eden et al, 2010) and dephosphorylates signal transducing adapter molecule 2, an endosomal protein colocalizing with clathrin and involved in the sorting of RTKs for lysosomal degradation (Stuible et al, 2010). Therefore, it appears likely that CBL, EPS15L, and ITSN2 are direct dephosphorylation targets of PTP1B.
Almost half of our identified candidates for PTP1B substrates have a central role in $B$ cells. We successfully validated all of them as PTP1B substrates by Co-IP experiments in combination with orthovanadate treatment. However, we did not target Ig $\beta$ directly as it forms a disulphide-linked heterodimer with Ig $\alpha$ (Siegers et al, 2006) and, thus, co-purifies with Ig $\alpha$. In addition to the phosphotyrosinedependent binding of BTK and PLCY2 to the PTP1B substrate trapping mutant, we also showed that BTK-pY223 and PLCY2-pY769 levels were increased in PTP1B KO cells after anti- $\lambda$ stimulation. Furthermore, we identified and validated VAV1 as PTP1B substrate in Ramos B cells. In MEF cells, pY141 of VAV3 was identified as PTP1B-dependent phosphosite (Mertins et al, 2008). Because VAV1 and VAV3 exhibit high sequence similarity ( 75\%) and redundant functions (Pearce et al, 2004), we propose that PTP1B may dephosphorylate VAV1 at pY142 which corresponds to PY141 in VAV3.

In this work, we demonstrated that PTP1B has a multitude of substrates in B cells. Thus, an important question is how PTP1B reaches its substrates in a cellular context?

PTP1B has a proline-rich region through which the interaction with $\mathrm{SH} 3$ domain-containing proteins is mediated. For example, the PTP1B-dependent dephosphorylation of breast cancer anti-estrogen resistance protein 1 (BCAR1) was shown to depend on the interaction of PTP1B with the SH3 domain of BCAR1 (Liu et al, 1996). Indeed, we found that about one third of the identified PTP1B substrates contain at least one SH3-domain by which they potentially interact with PTP1B. Among these proteins was also GRB2, which contains a $\mathrm{SH} 2$ domain flanked by two SH3 domains. By Co-IP and immunoblot analysis we showed that GRB2 directly interacts with WT PTP1B. The binding of GRB2 to the PTP1B substrate trapping mutant was enhanced, but reduced upon orthovanadate treatment. This suggests that GRB2 interacts with PTP1B, independently of being a substrate of PTP1B.

Our data further showed that the interaction of PTP1B with Ig $\alpha$ and $C D 22$ requires its ER-targeting domain. This suggest that PTP1B plays an important role at the ER where it may prevent uncontrolled phosphorylation and activation of newly produced receptor components before they are assembled into protein complexes or membrane nano-domains that regulate their activity. Interestingly, the PTP1B-GRB2 interaction was also reduced in case of the truncated PTP1B trapping mutant that lost its location at the ER. Thus, GRB2 may connect CD22 to ER membrane-bound PTP1B. We showed that PTP1B dephosphorylates CD22 at pY807 in its intracellular domain. CD22 directly interacts with the adaptor protein GRB2, with GRB2 binding being dependent on CD22-pY807 (Poe et al, 2000; Vanshylla et al, 2018). Thus, it is tempting to speculate that PTP1B is recruited to CD22 via binding to GRB2. In this complex, PTP1B may also reach and dephosphorylate other substrates such as PLCY2, SYK, and BTK, which are now in close proximity.

in cell lysates and eluates by SDS-PAGE immunoblotting using anti-Myc antibody. The FLAG-tagged PTP1B trapping mutant was detected using anti-FLAG antibody. Coimmunoprecipitation of GRB2 was detected using a GRB2 antibody ( $n=1)$. (D) Quantification of immunoblot data from (C). Anti-FLAG signals (FLAG-PTP1B-D181A-Y46F) were normalized to anti-myc signals (myc-tagged CD22 and site mutants thereof). A t test was performed testing each mutant against the control and $P$-values were calculated $(n=3)$. Error bars represent the SEM. (E) Phosphatase assay in FLAG-PTP1B-inducible Ramos cells. Myc-tagged CD22 was immunoprecipitated from untreated or anti- $\lambda$ stimulated cells, with or without the expression of FLAG-PTP1B. Phosphorylation levels of CD22-Y807 were monitored by immunoblot. (F) Quantification of immunoblots from (E). CD22-pY807 signals were normalized to the signals for immunoprecipitated CD22-myc. A t test was performed and $P$-values were calculated ( $n=3$ ). Error bars represent the SEM.

Source data are available online for this figure. 
Dephosphorylation of CD22-pY807 will then provide a switch for releasing GRB2 and, thereby, protecting CD22 and other substrates from further dephosphorylation by PTP1B.

\section{Materials and Methods}

\section{Cell culture and cell lines}

Ramos B cells were cultivated in Roswell Park Memorial Institute (RPMI) 1640 Medium with stable L-glutamine (PAN Biotech) supplemented with $10 \%$ heat-inactivated FCS (Biochrom). For SILAC experiments, cells were cultured for at least 6 d in RPMI 1640 Medium supplemented with $10 \%$ dialyzed FCS (Sigma-Aldrich), containing either "heavy" lysine (40 $\mathrm{g} / \mathrm{l} ; 13 \mathrm{C6}, 15 \mathrm{~N} 2)$ and arginine (80 g/l; 13C6, 15N4), "medium" lysine (40 g/l; 4,4,5,5-D4) and arginine (80 g/l; 13C6), or "light" lysine (40 g/l) and arginine (80 g/l). To prevent the conversion of arginine to proline, $80 \mathrm{~g} / \mathrm{l}$ proline was added. All stable isotope-labelled amino acids were purchased from Cambridge Isotope Laboratories. The "light" versions of the SILAC amino acids were obtained from Sigma-Aldrich.

For inducible expression of FLAG-PTP1B, FLAG-PTP1B D181A Y46F, or FLAG-PTP1B D181A-Y46F $\triangle 406-435$ (missing 30 amino acids at the C-terminus) PTP1B KO Ramos were transfected with a doxycycline inducible tet-on vector and exposed to $2 \mu \mathrm{g} / \mathrm{ml}$ doxycycline (Calbiochem, Merck Millipore) 24-48 h before the experiment. Platinum E or Phoenix cells were used as packaging cell lines to generate virus particles. Cells were transfected with the corresponding plasmids using polyethylenimine ( $1 \mu \mathrm{g} / \mu \mathrm{l}$ [pH 7]; Polysciences) in a 1:3 ratio. The virus supernatants were harvested 48 and $72 \mathrm{~h}$ after transfection. For each plasmid, $2 \times 10^{6}$ Ramos B cells were infected with $500 \mu$ l virus supernatant for $3 \times 60$ min centrifuging at $100 \mathrm{~g}$ at $37^{\circ} \mathrm{C}$. Cell sorting was performed on the Bio-Rad Sorter S3e instrument with the Software ProSort 1.5 (Bio-Rad).

\section{Molecular cloning}

Primers and plasmids used in this study are listed in Table S10. pMIG-FLAG-PTP1B-D181A (Medgyesi et al, 2014) was used as a PCR template to obtain PMIG-FLAG-PTP1B-D181A-Y46F. Two flanking PCR fragments containing the point mutation from tyrosine to phenylalanine and spanning two EcoRI restriction sites were amplified and joined by PCR. The fused product was ligated into the pJet1.2 cloning vector (Thermo Fisher Scientific). EcoRI (New England Biolabs) was used for restriction digest, to linearize the pMIG-FLAGPTP1B-D181A vector, and to release the point mutation containing insert from pJET1.2. Both were ligated to obtain PMIG-FLAG-PTP1BD181A-Y46F.

PMIG-FLAG-PTP1B-Y46F $\triangle 406-435$ was cloned using pMIG-FLAGPTP1B-D181A-Y46F as PCR template. The reverse primer was designed to introduce a stop codon and an EcoRI restriction site. The product was ligated into pJet1.2, followed by EcoRI restriction cloning as described above.

GST-tagged PTP1B for the peptide-based phosphatase assay was cloned using PMIG-FLAG-PTP1B. The vector was digested with BamHI and EcoRI and PTP1B was ligated into pGEX-2T (GE Healthcare).
The doxycycline-inducible tet-on system comprises two vectors: SH582 and SH461MK-EV-GFP (Haug et al, 2015) and was obtained from TET Systems $\mathrm{GmbH} \&$ Co. KG. The antibiotic resistance of the first vector was changed to bleomycin (SH582-bleomycin) by Gibson cloning (Gibson et al, 2009) using SH582 as backbone and pGIPZ (Open Biosystems) for the bleomycin template. The second vector, pSH461MK-EV-GFP, was used as a control plasmid. Alternatively, the CDNA of FLAG-PTP1B, FLAG-PTP1B-D181A-Y46F, and FLAG-PTP1BD181A-Y46F $\triangle 406-435$ was integrated into pSH461MK-EV-GFP using the corresponding PMIG-vector as a template for Gibson cloning. Carboxy-terminally Myc-tagged CD22 was generated by Gibson cloning. For this, pMSCV-TwinStrep_CD22 human (kind gift of AG Nitschke) (Meyer et al, 2020) and pMICD8 mb-1 FLAG (Infantino et al, 2010) were used as templates to amplify human CD22 and the pMICD8 backbone (Herzog \& Jumaa, 2007), respectively. The Myctag was introduced by the Gibson primers. The CD22 point mutations from tyrosine to phenylalanine were introduced by site-directed mutagenesis according to the QuikChange method (Agilent Technologies).

\section{CRISPR/Cas9-mediated PTP1B gene deficiency}

To generate PTP1B KO cells, a targeted DNA double strand break was introduced using two guide RNAs, creating offset nicks with the Cas9n D10A nickase mutant of Streptococcus pyogenes (Cong et al, 2013). The vectors pX461 (Addgene) and C250 (derived from pX461, GFP replaced with dsRED [Lindner et al, 2017]) were digested with BbsI (NEB) and ligated with the following annealed oligomers:

5'-CACCGTCCATCTCCATGACGGGCCA-3' and 5'AAACTGGCCCGTCATGGAGATGGAC-3' (first nick), 5'-CACCGATCGACAAGTCCGGGAGCT$3^{\prime}$ and 5'AAACAGCTCCCGGACTTGTCGATC-3' (second nick). Ramos B cells were electroporated to transfer $3 \mu \mathrm{g}$ DNA of each purified plasmid (1,350 V, 30 ms, 1 pulse, Neon Transfection System [Invitrogen]). Expression of the gRNAs was monitored by flow cytometry (GFP and dsRED expression).

\section{Calcium measurements}

For calcium measurements, $1 \times 10^{6}$ cells of each condition (Ramos cells treated with DMSO or with $0.5 \mu \mathrm{g} / \mathrm{ml}$ doxycycline for 2-3 d) were combined in a 1:1 ratio and loaded with $5 \mu \mathrm{g} / \mathrm{ml}$ Indo-1 (Molecular Probes, AAT Bioquest) and $0.04 \%$ of pluronic F-127 (AAT Bioquest) in RPMI containing 1\% FCS. Cells were incubated for $45 \mathrm{~min}$ at $37^{\circ} \mathrm{C}$ in the dark, washed twice with PBS and resuspended in $500 \mu \mathrm{l} \mathrm{serum}$-free RPMI. Cells were incubated for $10 \mathrm{~min}$ at $37^{\circ} \mathrm{C}$ immediately before the calcium flux analysis on an LSR Fortessa instrument (Becton Dickinson) using the following parameters: Indo-1 "bound" 355 nm, BP 405/10, and "unbound" 355 nm, 445LP, BP 520/35; GFP 488 nm, BP 530/30. A baseline of $30 \mathrm{~s}$ was recorded before stimulation with $1 \mu \mathrm{g} / \mathrm{ml}$ anti- $\lambda$ antibody (Southern Biotech). Flow cytometry standard files were analyzed using Flowjo software for Windows, version 10.6.0 (Becton Dickinson) and its integrated kinetics tool. To quantify differences in the calcium flux curves of PTP1B KO versus GFP or FLAG-PTP1B, a five point adjacent-averaging smoothing was performed using OriginPro 2017 (OriginLab Corporation). An average of the first $30 \mathrm{~s}$ of the calcium measurement was calculated and used to normalize the measured 
values to the baseline. At time point $300 \mathrm{~s}$ the difference in the Indo-1 (bound/unbound) ratio was calculated between the curves of each sample. For the quantification of the calcium flux in the experiments with the different trapping mutants, the curves were baseline corrected as described above and the area under the curve of each sample was calculated.

\section{Immunofluorescence microscopy}

To study the subcellular localization of FLAG PTP1B WT, FLAG-PTP1B D181A Y46F, or FLAG-PTP1B D181A-Y46F $\Delta 406-435$, doxycyclineinducible Ramos B cells were used. $2 \times 10^{6}$ cells were resuspended in $1 \mathrm{ml}$ serum-free RPMI. 400,000 cells were seeded on each cover slip and fixed with ice-cold methanol/acetone (1:1, vol/vol) for 10 min at $-20^{\circ} \mathrm{C}$, followed by washing with $1 \times$ ice-cold PBS. Permeabilized cells were blocked with $10 \%$ normal goat serum and $1 \%$ BSA in PBS for 40 min at RT. Subsequently, the cover slips were washed in $0.02 \%$ PBS-Tween 20 detergent (PBS-T), and cells were incubated with mouse anti-PTP1B FG6, Merck Millipore (1:100 in PBS$\mathrm{T})$, and rabbit anti-calnexin, Abcam (1:1,000 in PBS-T) for $2 \mathrm{~h}$ at RT. After washing $(3 \times)$ with $0.02 \%$ PBS-T, samples were incubated with goat anti-mouse AlexaFluor 594 (1:200 in PBS-T) and goat antirabbit AlexaFluor 488 antibodies (1:200 in PBS-T) for $2 \mathrm{~h}$ at RT. Cell nuclei were stained with DAPI (1:1,000) for 2 min at RT. For mounting, ProLongGold (Life Technologies) was used. Images were obtained using a commercial gated stimulated emission depletion (STED) microscope (TCS SP8 STED 3x) with an HC PL APO CS2 40×/1.30 oil objective (Leica Microsystems). Detection of DAPI, AlexaFluor 488, and 594 fluorophores was performed in sequential acquisition mode using photomultiplier tubes and hybrid detectors in the standard mode and wavelengths ranges of $425-460,503-550$, and 601-649 nm, respectively.

\section{Proximity ligation assay}

PLAs were performed as previously described (Kläsener et al, 2014). To generate PLA-probes against specific targets, the following unlabelled antibodies were used: anti-PTP1B (MABS197, FG6-1G; Merck Millipore), anti-Ig (HM47; Novus Biologicals), anti-CD22 (RFB4; Southern Biotech), and anti-APLP2 (15041-1-AP; Proteintech Group). The buffer of the anti-PTP1B antibody was exchanged to PBS-T using a Microcon-30 kD centrifugal filter unit (MRCF0R030; Merck Millipore) according to the manufacturer's instructions. Anti-Iga Fab fragment was prepared with Pierce Fab Micro preparation kit (Thermo Fisher Scientific) using immobilized papain according to the manufacturer's protocol. After desalting (Zeba spin desalting columns; Thermo Fisher Scientific), all antibodies were coupled with PLA Probemaker Plus or Minus oligonucleotides (Sigma-Aldrich) to generate PLA-probes. For in situ PLA, Ramos cells were settled on polytetrafluoroethylene slides (Thermo Fisher Scientific) for $30 \mathrm{~min}$ at $37^{\circ} \mathrm{C}$. Cells were fixed for 20 min with $4 \%$ paraformaldehyde, permeabilized with $0.5 \%$ saponin for $30 \mathrm{~min}$ at RT, and blocked for 30 min with blocking buffer $(25 \mu \mathrm{g} / \mathrm{ml}$ sonicated salmon sperm DNA, $250 \mu \mathrm{g} / \mathrm{ml}$ BSA, 1 M glycine). PLA was performed with Duolink In Situ Orange (Sigma-Aldrich). Samples were directly mounted on slides with DAPI Fluoromount-G (Southern
Biotech) to visualize the PLA signals in relationship to the nuclei. Microscopic images were acquired with a Leica DMi8 microscope, 63 oil objective (Leica-microsystems). For each experiment, a minimum of 1,000 Ramos B cells from several images were analyzed with CellProfiler-3.0.0 (CellProfiler.org). Raw data were exported to Prism 7 (GraphPad). For each sample, the mean PLA signal count per cell was calculated from the corresponding images and the statistical significance was determined using the Mann-Whitney test.

\section{Purification of recombinant PTP1B}

GST-PTP1B expression in BL21 competent Escherichia coli (NEB) was induced by the addition of $0.2 \mathrm{mM}$ IPTG. After incubation at $37^{\circ} \mathrm{C}$ for $3 \mathrm{~h}$, bacteria were pelleted at 7,000g for $10 \mathrm{~min}$. Cell lysis was performed for $1 \mathrm{~h}$ on ice in PBS supplemented with $10 \mathrm{mM} \mathrm{MgCl}$, $1 \mathrm{mM}$ PMSF, $0.1 \mathrm{mg} / \mathrm{ml}$ lysozyme and $5 \mathrm{U} / \mathrm{ml}$ benzonase and completed by $10 \mathrm{~min}$ sonification on ice. The lysate was cleared by centrifugation for $40 \mathrm{~min}$ at 25,000 $\mathrm{g}$ at $4^{\circ} \mathrm{C}$. GST-PTP1B was purified with a $1 \mathrm{ml}$ GSTrap FF Column on an ÄKTA pure purification system (GE Healthcare GmbH) using a binary buffer system (buffer A: $50 \mathrm{mM}$ Hepes, pH 8.5, 150 mM NaCl; buffer B: 50 mM Hepes, pH 8.0, 10 mM reduced glutathione). The lysate was applied at a flow rate of $1 \mathrm{ml} /$ min. A linear gradient from 0 to $100 \%$ buffer B in 10 column volumes was applied to elute the sample. The eluate was collected in $0.5 \mathrm{ml}$ fractions. PTP1B-containing fractions were pooled and supplemented with $20 \%$ glycerol.

\section{Materials for peptide synthesis and purification}

All amino acids and resins were purchased from Novabiochem (Merck Millipore) and Carbosynth. All other synthetic reagents were obtained from Novabiochem, Sigma-Aldrich, or Carl Roth. Peptide synthesis was performed on a MultiPep RSi peptide synthesizer (Intavis Bioanalytical Instruments AG). Peptides were purified using a 1260 Infinity II preparative HPLC system (Agilent Technologies) with a VP 250/10 NUCLEODUR 100-5 C18 ec column (MachereyNagel) running a general gradient of $10-70 \%$ acetonitrile (ACN) in $\mathrm{H}_{2} \mathrm{O}$ and validated using a 1260 Infinity I HPLC System coupled to a 6120 Quadrupole LC-MS (Agilent Technologies) with an EC 250/4 NUCLEODUR 100-5 C18 ec column (Macherey-Nagel) with a general gradient of $10-90 \% \mathrm{ACN}$ in $\mathrm{H}_{2} \mathrm{O}$, and additionally a Microflex LT MALDI (Bruker) was used for the analysis.

\section{Peptide synthesis}

Peptides were synthesized following fluorenylmethyloxycarbonyl(Fmoc) solid-phase peptide synthesis (Table S10). Pre-coupled, tertbutyloxycarbonyl-protected chlorotrityl resins were used for all peptides. Resins were swollen for 20 min in N,N-dimethylformamide (DMF) before synthesis in the peptide synthesizer. One round of synthesis was carried out for each amino acid in the peptide sequence. A synthetic round consisted of double coupling to an Fmoc and side chain-protected amino acid residue, followed by a capping step with acetic anhydride $\left(\mathrm{AC}_{2} \mathrm{O}\right)$, and then removal of the Fmoc group with piperidine in the preparation for the next round of coupling. Coupling reactions were carried out by adding Fmoc-protected amino acids ( $4 \mathrm{eq}$ ), O-benzotriazole- $\mathrm{N}, \mathrm{N}, \mathrm{N}^{\prime}, \mathrm{N}^{\prime}$-tetramethyl-uronium 
hexafluorophosphate (HBTU) (4 eq), Nhydroxybenzotriazolehydrate (HOBt) (4 eq), and N-methylmorpholine (NMM) to the resin in $1 \mathrm{ml} \mathrm{DMF}$ and reacting for 30-45 min. Capping was done by reaction with a $1 \mathrm{ml}$ solution of $5 \% \mathrm{AC}_{2} \mathrm{O}$ and $5 \%$ 2,6-Lutidine in DMF for 5 min. Fmocdeprotection was achieved by addition of a $20 \%$ piperidine in DMF solution to the resin, first for $3 \mathrm{~min}$, then again for $8 \mathrm{~min}$. Resins were washed with DMF between each step. After Fmoc removal of the final amino acid residue to reveal the free $\mathrm{N}$ terminus, peptides and libraries were removed from the resin and fully deprotected in one step by shaking overnight in cleavage cocktail (95\% TFA, 2.5\% triisopropylsilane [TIPS], and $2.5 \% \mathrm{H}_{2} \mathrm{O}$ ). Peptides were precipitated in cold ether $\left(-20^{\circ} \mathrm{C}\right)$ and collected by centrifugation. Peptides were then validated by MALDI and HPLC-MS, and purified by preparative HPLC.

\section{Detection of phosphate release from peptides}

The peptides were dissolved in $25 \%$ DMSO and $75 \% \mathrm{H}_{2} \mathrm{O}$ at $7.5 \mathrm{mM}$ after purification. The EnzChek Phosphate Assay Kit (Thermo Fisher Scientific) was used to assess the kinetics/dynamics of the peptide dephosphorylation by recombinant PTP1B. The assay was carried out in a volume of $100 \mu$ reaction buffer ( $20 \mathrm{mM}$ Tris, $100 \mathrm{mM} \mathrm{NaCl}, 2$ mM DTT, $0.15 \mathrm{U}$ purine nucleoside phosphorylase, $0.2 \mathrm{mM}$ 2-amino6-mercapto-7-methylpurine riboside) containing indicated concentrations of peptide, and $20 \mathrm{nM}$ recombinant PTP1B. The change of absorbance was detected at $360 \mathrm{~nm}$ and $28^{\circ} \mathrm{C}$ on a Synergy $\mathrm{H} 1$ microplate reader. For analysis of enzyme kinetics, raw data were further analyzed in GraphPad Prism v6. Error bars represent SD of three replicates. Values were compared to a standard curve prepared by using the phosphate solution included in the kit and following the manufacturer's instructions.

\section{B cell stimulation and cell lysis}

For stimulation of Ramos B cells, $1 \times 10^{7}$ cells were starved for 30 min at $37^{\circ} \mathrm{C}$ in $1 \mathrm{ml} \mathrm{FCS}$-free RPMl and then treated with $10 \mu \mathrm{g} / \mathrm{ml}$ anti- $\lambda$ antibody (Southern Biotech) under shaking at $750 \mathrm{rpm}$ and $37^{\circ} \mathrm{C}$ (ThermoMixer, Eppendorf) for the indicated time points. To stop the reaction, cells were spun down for $10 \mathrm{~s}$ at $21,000 \mathrm{~g}$, the supernatant was removed and the cell pellet was immediately frozen in liquid nitrogen. Cell lysis was performed with modified RIPA buffer (1× PBS, $1 \%$ Nonidet $\mathrm{P}-40,0.1 \%$ SDS, and $0.5 \%$ sodium deoxycholate), supplemented with $2 \mathrm{mM}$ sodium orthovanadate, $10 \mathrm{mM}$ sodium pyrophosphate, $9.5 \mathrm{mM}$ sodium fluoride, $10 \mathrm{mM} \beta$-glycerophosphate, and $1 \times$ EDTA-free protease inhibitor cocktail (Roche) before lysis.

\section{Co-immunoprecipitation}

$2 \times 10^{8}$ cells were lysed for $25 \mathrm{~min}$ in $5 \mathrm{ml}$ ice-cold lysis buffer $(50 \mathrm{mM}$ Tris- $\mathrm{HCl}, \mathrm{pH}$ 8.5, $137.5 \mathrm{mM} \mathrm{NaCl}, 1 \mathrm{mM}$ EDTA, 1\% Triton X-100, $1 \mathrm{mM}$ sodium fluoride, and EDTA-free protease inhibitor cocktail [Roche]). $1 \mathrm{mM}$ orthovanadate was added to the lysis buffer to prevent binding of substrates to the PTP1B trapping mutant. Lysates were cleared by centrifugation for $10 \mathrm{~min}$ at 4,969g. A small aliquot was taken for Western blot analysis of the lysate. The remaining sample was mixed with $40 \mu \mathrm{L}$ FLAG M2 magnetic beads (Sigma-Aldrich). The samples were incubated for $2-4 \mathrm{~h}$ on a rotating wheel at $8^{\circ} \mathrm{C}$, washed $3 \times$ with $1 \mathrm{ml}$ lysis buffer and eluted with $40 \mu \mathrm{l} 1 \times$ sample buffer (10\% glycerol, 1\% $\beta$-mercaptoethanol, 2\% SDS, $50 \mathrm{mM}$ Tris- $\mathrm{HCl}, \mathrm{pH} 6.8$, and $0.004 \%$ bromophenol blue).

For MS analysis, the proteins were eluted with $40 \mu \mathrm{l} 3 \times$ FLAG peptide (Sigma-Aldrich) and collected by centrifugation for $30 \mathrm{~min}$ at $100 \mathrm{~g}$.

For the phosphatase assay, Ramos B cells stably expressing CD22-Myc were used. Expression of FLAG-PTP1B was induced 18-24 $\mathrm{h}$ before the assay by the addition of doxycycline as indicated. Cells were stimulated with $10 \mu \mathrm{g} / \mathrm{ml}$ anti- $\lambda$ for 3 min at $37^{\circ} \mathrm{C}$ or left serum starved and lysed in $50 \mathrm{mM}$ Tris- $\mathrm{HCl}$ pH 8.0, 1\% Triton X-100, $137.5 \mathrm{mM} \mathrm{NaCl}$, supplemented with $2 \mathrm{mM}$ sodium orthovanadate, $10 \mathrm{mM}$ sodium pyrophosphate, $1 \mathrm{mM}$ sodium fluoride, $10 \mathrm{mM} \beta$ glycerolphosphate, and $1 \times$ protease inhibitor cocktail (Roche). For immunoprecipitation, Pierce anti-c-Myc magnetic beads (Thermo Fisher Scientific) were used.

\section{In-gel protein digestion}

Co-IP samples were loaded and separated on a 4-20\% Tris-Glycine gel (Invitrogen). Proteins were stained with colloidal Coomassie Brilliant Blue G-250. In gel-digestion of proteins was performed essentially as described before (Schwarz et al, 2015) with the exception that protein thiols were reduced in $5 \mathrm{mM}$ tris (2-chloroethyl) phosphine (TCEP) for $10 \mathrm{~min}$ at $60^{\circ} \mathrm{C}$ and alkylated in $100 \mathrm{mM}$ 2-chloroacetamide (CAM) for $15 \mathrm{~min}$ at $37^{\circ} \mathrm{C}$.

\section{Western blot analysis}

FLAG-tagged PTP1B was detected with anti-FLAG antibody (20543-1AP [Proteintech]). PTP1B was detected with anti-PTP1B clone FG6-1G (MABS197; Merck Millipore) or with clone H-135 (sc-14021) from Santa Cruz Biotechnology to detect specifically the C-terminal part. Anti-CD22 antibody was purchased from Novus Biological (clone 2H1C4), anti-CD79A (Ig $\alpha$; clone HM47) and anti-SYK (4D10.2) from BioLegend. The antibodies directed against; BTK-pY223, \#5082; PLCY1, \#5690; PLCY2, \#3872; PLCY2-pY759, \#3874; SYK-p525/526, \#2710; ERK, \#4695; ERK-pT202/Y204, \#4370; GRB2, \#3972; and GAPDH, \#2118 were all from Cell Signaling. Anti-phospho-CD22-pY807 (ab32040) antibody was purchased from Abcam. Anti-Myc-tag (66004-1-Ig), anti-APLP2 (15041-1-AP) and anti-CBL (25818-1-AP) were from Proteintech. Anti-BTK (sC-1107) and anti-BCAP (AF4857) antibodies were obtained from Santa Cruz Biotechnology and R\&D Systems Inc., respectively. All antibodies were used according to the manufacturer's instructions. For imaging, a ChemoCam (Intas) equipped with a full-frame 3.2 megapixel Kodak KAF-3200ME camera was used. Western blot signals were quantified with Quantity One 4.6.9 (BioRad). For statistical analysis, two-sample $t$ tests were performed over three replicates using Microsoft Excel 2013. Error bars represent the SEM.

\section{Sample preparation for quantitative proteome analysis}

Ramos B cells were harvested and washed three times with ice-cold 1× PBS. Cells were lysed in $30 \mathrm{mM}$ Tris base with $7 \mathrm{M}$ urea and $2 \mathrm{M}$ thiourea, $\mathrm{pH} 8.5$, supplemented with protease inhibitor (Roche). To aid cell lyses and to sheer DNA, cells were sonicated on ice at least twice with an ultrasonic homogenizer for $10 \mathrm{~s}$ at $90 \%$ of maximum 
power. Cell debris was removed by centrifugation for $20 \mathrm{~min}$ at $21,000 \mathrm{~g}$ and $4^{\circ} \mathrm{C}$. Samples containing $300 \mu \mathrm{g}$ of total protein were prepared and cysteine residues were reduced with $5 \mathrm{mM}$ TCEP for 20 $\mathrm{min}$ at RT and alkylated with $50 \mathrm{mM}$ CAM for $20 \mathrm{~min}$ at $37^{\circ} \mathrm{C}$. The reaction was quenched with $10 \mathrm{mM}$ DTT. Each sample was then diluted 1:1 with $50 \mathrm{mM}$ ammonium bicarbonate and digested with Lys-C (protein to enzyme ratio 1:100; FUJIFILM Wako Chemicals Europe $\mathrm{GmbH}$ ) for $4 \mathrm{~h}$ at $37^{\circ} \mathrm{C}$ Subsequently, the sample was diluted 1:1 with $50 \mathrm{mM}$ ammonium bicarbonate and proteins were digested with trypsin (protein to enzyme ratio 1:50; Promega) overnight at $37^{\circ} \mathrm{C}$. The reaction was stopped by acidification with TFA ( $1 \%$ [vol/ vol] final concentration) and the peptide sample was desalted using a Empore Solid Phase Extraction cartridge (3M Bioanalytical Technologies). The cartridge was washed with $1 \mathrm{ml}$ methanol followed by $1 \mathrm{ml} 70 \%$ ACN with $0.1 \%$ TFA. The column was conditioned twice with $0.5 \mathrm{ml} 0.1 \%$ TFA before the sample was applied. Peptides were washed with $0.5 \mathrm{ml} 0.1 \%$ TFA and eluted with $0.5 \mathrm{ml} 70 \%$ ACN. Peptides were freeze-dried overnight using an Alpha 1-2 LDplus system (Christ).

\section{High-pH reversed-phase fractionation}

High-pH reversed-phase fractionation was performed on an Ultimate 3000 HPLC system equipped with a Gemini-NX 3 um C18 $110 \AA$ column (Phenomenex). The desalted sample was taken up in $300 \mu \mathrm{l}$ of $10 \mathrm{mM} \mathrm{NH}_{4} \mathrm{OH}$ (solvent A). A constant flow rate of $0.2 \mathrm{ml} / \mathrm{min}$ was applied and peptides were loaded onto the column at $1 \%$ solvent $B$ (10 $\mathrm{mM} \mathrm{NH}_{4} \mathrm{OH}$ in $90 \% \mathrm{ACN}$ ) for 5 min. Peptides were separated applying a linear gradient from $1 \%$ B to $61 \%$ B in 55 min, followed by an increase to $78 \%$ B in $2 \mathrm{~min}$, a decrease to $1 \%$ B in $5 \mathrm{~min}$, and reequilibration of the column for $16 \mathrm{~min}$. Fractions were collected every $43 \mathrm{~s}$, starting at $1.5 \mathrm{~min}$ until $70 \mathrm{~min}$. Of the collected 96 fractions, every $32^{\text {nd }}$ fraction was pooled.

\section{High-performance liquid chromatography and mass spectrometry}

Reversed-phase HPLC was performed on an UltimateTM 3000 rapid separation liquid chromatography (RSLC) nano system (Thermo Fisher Scientific) equipped with two PepMap C18 $\mu$-precolumn (0.3 $5 \mathrm{~mm}, 5 \mu \mathrm{m}, 300 \AA$; Thermo Fisher Scientific) and an Acclaim PepMap analytical column $(75 \mu \mathrm{m} \times 250 \mathrm{~mm}, 3 \mu \mathrm{m}, 100 \AA$; Thermo Fisher Scientific).

For LC-MS analysis of affinity-purified samples (SILAC CO-IP), the RSLC system was coupled to a Velos Orbitrap Elite instrument (Thermo Fisher Scientific). A binary solvent system was used with solvent A consisting of $0.1 \%$ formic acid (FA) and $4 \%$ DMSO and solvent B consisting of $48 \%$ methanol, $30 \%$ ACN, $0.1 \%$ FA, and $4 \%$ DMSO. Peptides from SILAC Co-IPS were washed and concentrated for 5 min with $0.1 \%$ TFA on the pre-column. The gradient applied for peptide separation on the analytical column (flow rate of $0.250 \mu \mathrm{l}$ / min) was as follows: 1-65\% solvent B in 50 min, increased to $95 \%$ B in $5 \mathrm{~min}$, and $5 \mathrm{~min}$ at $95 \% \mathrm{~B}$, followed by a decrease to $1 \% \mathrm{~B}$ in $1 \mathrm{~min}$. Subsequently, the column was re-equilibrated for 14 min with $1 \%$ B. The Velos Orbitrap Elite instrument was operated with the following parameter settings: spray voltage, $1.5 \mathrm{kV}$; capillary voltage, $200 \mathrm{~V}$; automatic gain control (AGC), $1 \times 10^{6}$ ions; max. ion time (IT), $200 \mathrm{~ms}$.
Full scans were acquired in the orbitrap with a mass range of $\mathrm{m} / \mathrm{z}$ $370-1,700$ and a resolution (R) of 60,000 at $\mathrm{m} / \mathrm{z} 400$. A TOP15 method was applied for collision-induced dissociation (CID) of multiply charged peptides performed in the linear ion trap with a normalized collision energy of $35 \%$ and an activation q of 0.25 . The AGC for CID was set to 5,000, the max. IT was $150 \mathrm{~ms}$ and the dynamic exclusion time (DE) for previously selected precursor ions was $45 \mathrm{~s}$.

For quantitative proteome analysis, the RSLC system was coupled to a Q Exactive Plus instrument (Thermo Fisher Scientific). A solvent system consisting of $0.1 \%$ FA (solvent A) and $86 \%$ ACN, $0.1 \%$ FA (solvent B) was used for peptide separation. The RSLC was operated with a flow rate of $0.250 \mu \mathrm{l} / \mathrm{min}$ for the analytical column. The gradient was 4-40\% B in 50 min, followed by $40-95 \%$ B in 5 min. After 5 min at 95\%, the column was re-equilibrated for 15 min with $4 \% \mathrm{~B}$. Full scans were acquired in the orbitrap in a mass range of $\mathrm{m} / \mathrm{z}$ 375-1,700 with the following parameters: R, 70,000 at $\mathrm{m} / \mathrm{z} 200$; $\mathrm{AGC}, 3 \times 10^{6}$ ions; max. IT, $60 \mathrm{~ms}$. For MS/MS experiments employing higher-energy collisional dissociation (HCD), a TOP12 method was used and parameters were as follows: normalized collision energy, 28\%; DE 45 s; AGC, 5,000; max. IT, 120 ms.

\section{Bioinformatics}

Raw data were searched against the Uniprot Human Proteome set (release 2017_06, 93,591 protein entries) using MaxQuant 1.5.5.1 (for SILAC Co-IP and for global proteome analysis in Ramos B cells) or version 1.5.4.0 (quantitative proteome analysis of PTP1B KO Ramos B cells) with its integrated search engine Andromeda (Cox \& Mann, 2008; Cox et al, 2011). The species was restricted to homo sapiens as Ramos B cells are of human origin. MaxQuant default settings were used unless stated otherwise. Database searches were conducted with trypsin (for SILAC Co-IPS) or with trypsin and Lys-C (for quantitative proteome analysis) as proteolytic enzymes, a maximum number of three missed cleavages, and mass tolerance of 4.5 ppm for precursor and $0.5 \mathrm{D}$ (CID data) or $20 \mathrm{ppm}$ (HCD data) for fragment ions. For the quantitative proteome analysis of PTP1B KO cells, two missed cleavages were allowed. Carbamidomethylation of cysteine residues was set as fixed modification, and oxidation of methionine, $\mathrm{N}$-terminal acetylation were applied as variable modifications and additionally serine/threonine/tyrosine phosphorylation for SILAC Co-IPS and for global proteome analysis of Ramos B cells. The minimum number of unique peptides was set to one. A false discovery rate of $1 \%$ was applied to both peptide and protein lists. For global proteome analysis, "label-free" and "iBAQ" quantification as well as "match between runs" were enabled with default settings. For SILAC Co-IPS, the multiplicity was set to three and "Requantify" was enabled. The maximum number of labelled amino acids was set to four. For the quantitative proteome analysis of PTP1B KO cells, multiplicity was set to two, the maximum number of labelled amino acids to three and "Requantify" was enabled. For protein quantification, "razer and unique peptides" were used with a minimum ratio count of two. For the determination of significantly enriched PTP1B substrate candidates, non-normalized SILAC ratios were $\log _{10}$-transformed and a mean $\log _{10}$ ratio over at least two out of three replicates was calculated. $P$-values were determined with a rightsided $t$ test in Perseus 1.5.5.3 (Tyanova et al, 2016). For GO and REACTOME pathway enrichment analysis, the Cytoscape 3.2.1 (Shannon 
et al, 2003) plugin ClueGO v2.5.1 (Bindea et al, 2009) was used. The global proteome dataset was divided into five quantiles with an equal number of protein groups according to their abundance rank. Enrichment of each quantile against the human reference set was analyzed for the GO domain "biological process" (BP) and for REACTOME pathways. A right-sided hypergeometric statistic test with Bonferroni step down false discovery rate correction at a significance level of 0.05 was used for P-value calculation. For network analysis of PTP1B substrate candidates identified by SILAC Co-IPS, the STRING application in Cytoscape 3.8.2 was used. For network analysis of the Ramos B cell proteome, the pathway was downloaded from NetPath (Kandasamy et al, 2010) and processed in Cytoscape 3. 2. 1. Enrichment of SH3 domain-containing proteins was calculated using the SMART database (Letunic et al, 2015; Letunic \& Bork, 2018). For the quantitative proteome analysis of PTP1B KO cells, normalized SILAC ratios were $\log _{2}$-transformed, a mean $\log _{2}$ ratio was calculated over three of four biological replicates and a two-sided $t$ test was applied to determine $P$-values. Extracted ion chromatograms of PTP1B peptide precursor ions were analyzed manually in Skyline 3.5.0 (Schilling et al, 2012).

\section{Protein copy number determination}

To determine protein copy numbers, the proteomic ruler plugin v.0.1.6 (available from http:/ / www.coxdocs.org/doku.php?id=perseus: user:plugins:store) for Perseus 1.5.5.3 was used (Tyanova et al, 2016). The applied settings were as follows: averaging mode, "same normalization for all columns"; detectability correction, number of detectable tryptic peptides; quantification, histones as proteomic ruler; ploidy of the cells, 1.9565 as Ramos B cells have 45 chromosomes. From the resulting copy numbers of three biological replicates, mean $\log _{10}$ copy numbers were calculated for protein groups with at least two valid values.

\section{RNA sequencing data analysis}

RNA sequencing raw files from Qian et al (2014) were downloaded from the sequence read archive with the following accession numbers: SRX753158 (replicate 1), SRX753159 (replicate 2) and SRX753160 (replicate 3). The quality of the raw data was assessed with FastQC v0.11.5 (https://www.bioinformatics.babraham.ac.uk/ projects/fastqc/). Raw reads had a length of 50 bases and were trimmed with BBDuk v.37.28 implemented into Geneious v11.0.5 (http://www.geneious.com [Kearse et al, 2012]) on both ends, if the quality of the base call was $<20$ phred score. Reads shorter than 45 bases were discarded. Remaining reads were mapped against $H$. sapiens GRCh38.p7 primary assembly with the Geneious RNA assembler using default settings at medium sensitivity and with the minimum mapping quality adjusted to $99.9 \%$ confidence. Expression levels (RPKM and TPM) were calculated for each replicate with Geneious v11.0.5. As a measure for transcript abundance, TPM was used (Wagner et al, 2012). TPM values for each replicate were $\log _{2}{ }^{-}$ transformed and mean $\log _{2}$ TPM values were calculated. Resulting data were binned into a histogram with Origin 2017 (OriginLab Corporation). A cut-off value of $\log _{2}$ TPM $=0$ was set for genes considered to be expressed. Entries with non-zero TPM values were mapped against the UniProt database according to their GeneID.
Non-mapping entries were further mapped according to their gene name to Uniprot identifiers. All mapped entries were considered as protein-coding genes.

\section{Data Availability}

All raw data and original MaxQuant result files have been deposited to the ProteomeXchange Consortium (http://proteomecentral. proteomexchange.org) via the PRIDE partner repository (PerezRiverol et al, 2019) with the dataset identifiers PXD024037 (largescale quantitative analysis of human Ramos B cells), PXD024086 (quantitative PTP1B substrate trapping) and PXD024038 (quantitative analysis of Ramos $B$ cells versus Ramos B cells with PTP1B KO).

\section{Supplementary Information}

Supplementary Information is available at https://doi.org/10.26508/lsa. 202101084.

\section{Acknowledgements}

We thank the PRIDE team for data deposition to the ProteomeXchange Consortium, Bettina Knapp for technical assistance in LC-MS analysis, and Lars Nitschke and Sarah Meyer for scientific discussions. Work included in this study has been performed in partial fulfillment of the requirements for the doctoral theses of JJ Schwarz and T Kokot, and the master theses of $S$ Fotteler and L Grundmann at the University of Freiburg. This work was supported by the Deutsche Forschungsgemeinschaft (DFG, German Research Foundation) TRR 130 project P05 (to B Warscheid and M Reth) and project C02 (to B Warscheid), Project ID 403222702/SFB 1381 (to B Warscheid and M Köhn), FOR 2743 (to B Warscheid and M Köhn), as well as Germany's Excellence Strategy (CIBSS-EXC-2189-Project ID 390939984; to B Warscheid, M Reth, and $M$ Köhn) and the Excellence Initiative of the German federal and state governments (EXC 294, BIOSS; GSC-4, Spemann Graduate School; to B Warscheid, M Reth, and M Köhn).

\section{Author Contributions}

J Schwarz: data curation, formal analysis, validation, investigation, visualization, and writing-original draft, review, and editing.

L Grundmann: investigation.

T Kokot: investigation.

K Kläsener: investigation.

S Fotteler: investigation.

D Medgyesi: investigation.

M Köhn: resources, funding acquisition, and writing-review and editing. M Reth: conceptualization, resources, funding acquisition, and writingreview and editing.

B Warscheid: conceptualization, resources, supervision, funding acquisition, project administration, and writing-original draft, review, and editing.

\section{Conflict of Interest Statement}

The authors declare that they have no conflict of interest. 


\section{References}

Alonso A, Sasin J, Bottini N, Friedberg I, Friedberg I, Osterman A, Godzik A, Hunter T, Dixon J, Mustelin T (2004) Protein tyrosine phosphatases in the human genome. Cell 117: 699-711. doi:10.1016/j.cell.2004.05.018

Aoki Y, Isselbacher KJ, Pillai S (1994) Bruton tyrosine kinase is tyrosine phosphorylated and activated in pre-B lymphocytes and receptorligated B cells. Proc Natl Acad Sci U S A 91: 10606-10609. doi:10.1073/ pnas.91.22.10606

Banh RS, Iorio C, Marcotte R, Xu Y, Cojocari D, Rahman AA, Pawling J, Zhang W, Sinha A, Rose CM, et al (2016) PTP1B controls non-mitochondrial oxygen consumption by regulating RNF213 to promote tumour survival during hypoxia. Nat Cell Biol 18: 803-813. doi:10.1038/ncb3376

Bindea G, Mlecnik B, Hackl H, Charoentong P, Tosolini M, Kirilovsky A, Fridman WH, Pagès F, Trajanoski Z, Galon J (2009) ClueGO: A cytoscape plug-in to decipher functionally grouped gene ontology and pathway annotation networks. Bioinformatics 25: 1091-1093. doi:10.1093/ bioinformatics/btp101

Boelens J, Lust S, van Bockstaele F, van Gele M, Janssens A, Derycke L, Vanhoecke B, Philippé J, Bracke M, Offner F (2009) Steroid effects on ZAP-70 and SYK in relation to apoptosis in poor prognosis chronic lymphocytic leukemia. Leuk Res 33: 1335-1343. doi:10.1016/ j.leukres.2009.02.022

Boubekeur S, Boute N, Pagesy P, Zilberfarb V, Christeff N, Issad T (2011) A new highly efficient substrate-trapping mutant of protein tyrosine phosphatase 1B (PTP1B) reveals full autoactivation of the insulin receptor precursor. J Biol Chem 286: 19373-19380. doi:10.1074/ jbc.M111.222984

Chen J, Wang H, Xu WP, Wei SS, Li HJ, Mei YQ, Li YG, Wang YP (2016) Besides an ITIM/SHP-1-dependent pathway, CD22 collaborates with Grb2 and plasma membrane calcium-ATPase in an ITIM/SHP-1-independent pathway of attenuation of $\mathrm{Ca} 2+\mathrm{i}$ signal in B cells. Oncotarget 7 : 56129-56146. doi:10.18632/oncotarget.9794

Chong YP, Mulhern TD, Cheng HC (2005) C-terminal Src kinase (CSK) and CSKhomologous kinase (CHK): Endogenous negative regulators of Srcfamily protein kinases. Growth Factors 23: 233-244. doi:10.1080/ 08977190500178877

Cong L, Ran FA, Cox D, Lin S, Barretto R, Habib N, Hsu PD, Wu X, Jiang W, Marraffini LA, et al (2013) Multiplex genome engineering using CRISPR/ Cas systems. Science 339: 819-823. doi:10.1126/science.1231143

Cornall RJ, Cyster JG, Hibbs ML, Dunn AR, Otipoby KL, Clark EA, Goodnow CC (1998) Polygenic autoimmune traits: Lyn, CD22, and SHP-1 are limiting elements of a biochemical pathway regulating BCR signaling and selection. Immunity 8: 497-508. doi:10.1016/S1074-7613(00)80554-3

Cox J, Mann M (2008) MaxQuant enables high peptide identification rates, individualized p.p.b.-range mass accuracies and proteome-wide protein quantification. Nat Biotechnol 26: 1367-1372. doi:10.1038/nbt.1511

Cox J, Neuhauser N, Michalski A, Scheltema RA, Olsen JV, Mann M (2011) Andromeda: A peptide search engine integrated into the MaxQuant environment. J Proteome Res 10: 1794-1805. doi:10.1021/pr101065j

Cyster JG, Goodnow CC (1995) Protein tyrosine phosphatase 1C negatively regulates antigen receptor signaling in $\mathrm{B}$ lymphocytes and determines thresholds for negative selection. Immunity 2: 13-24. doi:10.1016/1074-7613(95)90075-6

Damle NP, Köhn M (2019) The human DEPhOsphorylation database DEPOD: 2019 update. Database (Oxford) 2019: baz133. doi:10.1093/database/ baz133

Delibrias CC, Floettmann JE, Rowe M, Fearon DT (1997) Downregulated expression of SHP-1 in Burkitt lymphomas and germinal center B lymphocytes. J Exp Med 186: 1575-1583. doi:10.1084/jem.186.9.1575

de Weers M, Brouns GS, Hinshelwood S, Kinnon C, Schuurman RK, Hendriks RW, Borst J (1994) B-cell antigen receptor stimulation activates the human Bruton's tyrosine kinase, which is deficient in X-linked agammaglobulinemia. J Biol Chem 269: 23857-23860. doi:10.1016/ S0021-9258(19)51014-6

Dubé N, Bourdeau A, Heinonen KM, Cheng A, Loy AL, Tremblay ML (2005) Genetic ablation of protein tyrosine phosphatase $1 B$ accelerates lymphomagenesis of p53-null mice through the regulation of B-cell development. Cancer Res 65: 10088-10095. doi:10.1158/00085472.CAN-05-1353

Dustin LB, Plas DR, Wong J, Hu YT, Soto C, Chan AC, Thomas ML (1999) Expression of dominant-negative src-homology domain 2-containing protein tyrosine phosphatase-1 results in increased Syk tyrosine kinase activity and B cell activation. / Immunol 162: 2717-2724. https:/ / www.jimmunol.org/content/162/5/2717

Eden ER, White IJ, Tsapara A, Futter CE (2010) Membrane contacts between endosomes and ER provide sites for PTP1B-epidermal growth factor receptor interaction. Nat Cell Biol 12: 267-272. doi:10.1038/ncb2026

Elchebly M, Payette P, Michaliszyn E, Cromlish W, Collins S, Loy AL, Normandin D, Cheng A, Himms-Hagen J, Chan CC, et al (1999) Increased insulin sensitivity and obesity resistance in mice lacking the protein tyrosine phosphatase-1B gene. Science 283: 1544-1548. doi:10.1126/ science.283.5407.1544

Fan G, Lin G, Lucito R, Tonks NK (2013) Protein-tyrosine phosphatase 1B antagonized signaling by insulin-like growth factor-1 receptor and kinase BRK/PTK6 in ovarian cancer cells. J Biol Chem 288: 24923-24934. doi:10.1074/jbc.M113.482737

Ferrari E, Tinti M, Costa S, Corallino S, Nardozza AP, Chatraryamontri A, Ceol A, Cesareni G, Castagnoli L (2011) Identification of new substrates of the protein-tyrosine phosphatase PTP1B by Bayesian integration of proteome evidence. J Biol Chem 286: 4173-4185. doi:10.1074/ jbc.M110.157420

Flint AJ, Tiganis T, Barford D, Tonks NK (1997) Development of "substratetrapping" mutants to identify physiological substrates of protein tyrosine phosphatases. Proc Natl Acad Sci U S A 94: 1680-1685. doi:10.1073/pnas.94.5.1680

Frangioni JV, Beahm PH, Shifrin V, Jost CA, Neel BG (1992) The nontransmembrane tyrosine phosphatase PTP-1B localizes to the endoplasmic reticulum via its 35 amino acid $C$-terminal sequence. Cell 68: 545-560. doi:10.1016/0092-8674(92)90190-n

Gibson DG, Young L, Chuang RY, Venter JC, Hutchison CA, Smith HO (2009) Enzymatic assembly of DNA molecules up to several hundred kilobases. Nat Methods 6: 343-345. doi:10.1038/nmeth.1318

Haj FG, Markova B, Klaman LD, Bohmer FD, Neel BG (2003) Regulation of receptor tyrosine kinase signaling by protein tyrosine phosphatase1B. J Biol Chem 278: 739-744. doi:10.1074/jbc.M210194200

Hata A, Sabe H, Kurosaki T, Takata M, Hanafusa H (1994) Functional analysis of Csk in signal transduction through the B-cell antigen receptor. Mol Cell Biol 14: 7306-7313. doi:10.1128/mcb.14.11.7306-7313.1994

Haug S, Schnerch D, Halbach S, Mastroianni J, Dumit VI, Follo M, Hasenburg A, Köhler M, Dierbach H, Herzog S, et al (2015) Metadherin exon 11 skipping variant enhances metastatic spread of ovarian cancer. Int J Cancer 136: 2328-2340. doi:10.1002/ijc.29289

Hebenstreit D, Fang M, Gu M, Charoensawan V, van Oudenaarden A, Teichmann SA (2011) RNA sequencing reveals two major classes of gene expression levels in metazoan cells. Mol Syst Biol 7: 497-499. doi:10.1038/msb.2011.28

Herzog S, Jumaa $\mathrm{H}$ (2007) The $\mathrm{N}$ terminus of the non-T cell activation linker (NTAL) confers inhibitory effects on pre-B cell differentiation. J Immunol 178: 2336-2343. doi:10.4049/jimmunol.178.4.2336

Hunter T (2000) Signaling: 2000 and beyond. Cell 100: 113-127. doi:10.1016/ S0092-8674(00)81688-8

Infantino S, Benz B, Waldmann T, Jung M, Schneider R, Reth M (2010) Arginine methylation of the $B$ cell antigen receptor promotes differentiation. J Exp Med 207: 711-719. doi:10.1084/jem.20091303 
Kandasamy K, Mohan SS, Raju R, Keerthikumar S, Kumar GS, Venugopal AK, Telikicherla D, Navarro JD, Mathivanan S, Pecquet C, et al (2010) NetPath: A public resource of curated signal transduction pathways. Genome Biol 11: R3-R9. doi:10.1186/gb-2010-11-1-r3

Katagiri T, Ogimoto M, Hasegawa K, Arimura Y, Mitomo K, Okada M, Clark MR, Mizuno K, Yakura H (1999) CD45 negatively regulates lyn activity by dephosphorylating both positive and negative regulatory tyrosine residues in immature B cells. I Immunol 163: 1321-1326. https:/ / www.jimmunol.org/content/163/3/1321

Kearse M, Moir R, Wilson A, Stones-Havas S, Cheung M, Sturrock S, Buxton S, Cooper A, Markowitz S, Duran C, et al (2012) Geneious basic: An integrated and extendable desktop software platform for the organization and analysis of sequence data. Bioinformatics 28 : 1647-1649. doi:10.1093/bioinformatics/bts199

Kessels HWHG, Ward AC, Schumacher TNM (2002) Specificity and affinity motifs for Grb2 SH2-ligand interactions. Proc Natl Acad Sci U S A 99: 8524-8529. doi:10.1073/pnas.142224499

Kläsener K, Maity PC, Hobeika E, Yang J, Reth M (2014) B cell activation involves nanoscale receptor reorganizations and inside-out signaling by Syk. Elife 3: e02069. doi:10.7554/eLife.02069

Law CL, Sidorenko SP, Chandran KA, Zhao Z, Shen SH, Fischer EH, Clark EA (1996) CD22 associates with protein tyrosine phosphatase 1C, Syk, and phospholipase C-gamma(1) upon B cell activation. J Exp Med 183: 547-560. doi:10.1084/jem.183.2.547

Letunic I, Bork P (2018) 20 years of the SMART protein domain annotation resource. Nucleic Acids Res 46: D493-D496. doi:10.1093/nar/gkx922

Letunic I, Doerks T, Bork P (2015) SMART: Recent updates, new developments and status in 2015. Nucleic Acids Res 43: D257-D260. doi:10.1093/nar/ gku949

Li X, Köhn M (2016) Prediction and verification of novel peptide targets of protein tyrosine phosphatase 1B. Bioorg Med Chem 24: 3255-3258. doi:10.1016/j.bmc.2016.03.030

Li X, Wilmanns M, Thornton J, Köhn M (2013) Elucidating human phosphatasesubstrate networks. Sci Signal 6: rs10. doi:10.1126/scisignal.2003203

Lindner SE, Lohmüller M, Kotkamp B, Schuler F, Knust Z, Villunger A, Herzog S (2017) The miR-15 family reinforces the transition from proliferation to differentiation in pre-B cells. EMBO Rep 18: 1604-1617. doi:10.15252/ embr.201643735

Liu F, Hill DE, Chernoff J (1996) Direct binding of the proline-rich region of protein tyrosine phosphatase $1 \mathrm{~B}$ to the Src homology 3 domain of p130(Cas). J Biol Chem 271: 31290-31295. doi:10.1074/jbc.271.49.31290

Lowenstein EJ, Daly RJ, Batzer AG, Li W, Margolis B, Lammers R, Ullrich A Skolnik EY, Bar-Sagi D, Schlessinger J (1992) The SH2 and SH3 domaincontaining protein GRB2 links receptor tyrosine kinases to ras signaling. Cell 70: 431-442. doi:10.1016/0092-8674(92)90167-b

Lu X, Malumbres R, Shields B, Jiang X, Sarosiek KA, Natkunam Y, Tiganis T, Lossos IS (2008) PTP1B is a negative regulator of interleukin 4-induced STAT6 signaling. Blood 112: 4098-4108. doi:10.1182/blood-2008-03148726

Martin-Granados C, Prescott AR, Le Sommer S, Klaska IP, Yu T, Muckersie E, Giuraniuc CV, Grant L, Delibegovic M, Forrester JV (2015) A key role for PTP1B in dendritic cell maturation, migration, and T cell activation. J Mol Cell Biol 7: 517-528. doi:10.1093/jmcb/mjv032

Matsumoto M, Oyamada K, Takahashi H, Sato T, Hatakeyama S, Nakayama KI (2009) Large-scale proteomic analysis of tyrosine-phosphorylation induced by T-cell receptor or B-cell receptor activation reveals new signaling pathways. Proteomics 9: 3549-3563. doi:10.1002/ pmic.200900011

Medgyesi D, Hobeika E, Biesen R, Kollert F, Taddeo A, Voll RE, Hiepe F, Reth M (2014) The protein tyrosine phosphatase PTP1B is a negative regulator of CD40 and BAFF-R signaling and controls B cell autoimmunity. J Exp Med 211: 427-440. doi:10.1084/jem.20131196
Mertins P, Eberl HC, Renkawitz J, Olsen JV, Tremblay ML, Mann M, Ullrich A, Daub H (2008) Investigation of protein-tyrosine phosphatase 1B function by quantitative proteomics. Mol Cell Proteomics 7: 1763-1777. doi:10.1074/mcp.M800196-MCP200

Meyer S), Böser A, Korn MA, Koller C, Bertocci B, Reimann L, Warscheid B, Nitschke L (2020) Cullin 3 is crucial for pro-B cell proliferation, interacts with CD22, and controls CD22 internalization on B cells. Immunol 204: 3360-3374. doi:10.4049/jimmunol.1900925

Müller J, Obermeier I, Wöhner M, Brandl C, Mrotzek S, Angermüller S, Maity PC, Reth M, Nitschke L (2013) CD22 ligand-binding and signaling domains reciprocally regulate B-cell Ca2+ signaling. Proc Natl Acad Sci U S A 110: 12402-12407. doi:10.1073/pnas.1304888110

Myers MP, Andersen JN, Cheng A, Tremblay ML, Horvath CM, Parisien JP, Salmeen A, Barford D, Tonks NK (2001) TYK2 and JAK2 are substrates of protein-tyrosine phosphatase 1B. J Biol Chem 276: 47771-47774. doi:10.1074/jbc.C100583200

Nicolussi A, D'Inzeo S, Capalbo C, Giannini G, Coppa A (2017) The role of peroxiredoxins in cancer. Mol Clin Oncol 6: 139-153. doi:10.3892/ mco.2017.1129

O'Donovan DS, MacFhearraigh S, Whitfield J, Swigart LB, Evan GI, Mc Gee MM (2013) Sequential Cdk1 and Plk1 phosphorylation of protein tyrosine phosphatase 1B promotes mitotic cell death. Cell Death Dis 4: e468. doi:10.1038/cddis.2012.208

Otipoby KL, Draves KE, Clark EA (2001) CD22 regulates B cell receptormediated signals via two domains that independently recruit Grb2 and SHP-1. J Biol Chem 276: 44315-44322. doi:10.1074/jbc.M105446200

Pani G, Kozlowski M, Cambier JC, Mills GB, Siminovitch KA (1995) Identification of the tyrosine phosphatase PTP1C as a B cell antigen receptorassociated protein involved in the regulation of B cell signaling. J Exp Med 181: 2077-2084. doi:10.1084/jem.181.6.2077

Pearce AC, Senis YA, Billadeau DD, Turner M, Watson SP, Vigorito E (2004) Vav1 and vav3 have critical but redundant roles in mediating platelet activation by collagen. J Biol Chem 279: 53955-53962. doi:10.1074/ jbc.M410355200

Perez-Riverol Y, Csordas A, Bai J, Bernal-Llinares M, Hewapathirana S, Kundu DJ, Inuganti A, Griss J, Mayer G, Eisenacher M, et al (2019) The PRIDE database and related tools and resources in 2019: Improving support for quantification data. Nucleic Acids Res 47: D442-D450. doi:10.1093/ nar/gky1106

Petro JB, Rahman SM, Ballard DW, Khan WN (2000) Bruton's tyrosine kinase is required for activation of IkappaB kinase and nuclear factor kappaB in response to B cell receptor engagement. J Exp Med 191: 1745-1754. doi:10.1084/jem.191.10.1745

Poe JC, Fujimoto M, Jansen PJ, Miller AS, Tedder TF (2000) CD22 forms a quaternary complex with SHIP, Grb2, and Shc. A pathway for regulation of $B$ lymphocyte antigen receptor-induced calcium flux. J Biol Chem 275: 17420-17427. doi:10.1074/jbc.M001892200

Qian J, Wang Q, Dose M, Pruett N, Kieffer-Kwon KR, Resch W, Liang G, Tang Z, Mathé E, Benner C, et al (2014) B cell super-enhancers and regulatory clusters recruit AID tumorigenic activity. Cell 159: 1524-1537. doi:10.1016/j.cell.2014.11.013

Rieckmann JC, Geiger R, Hornburg D, Wolf T, Kveler K, Jarrossay D, Sallusto F, Shen-Orr SS, Lanzavecchia A, Mann M, et al (2017) Social network architecture of human immune cells unveiled by quantitative proteomics. Nat Immunol 18: 583-593. doi:10.1038/ni.3693

Satpathy S, Wagner SA, Beli P, Gupta R, Kristiansen TA, Malinova D, Francavilla C, Tolar P, Bishop GA, Hostager BS, et al (2015) Systems-wide analysis of BCR signalosomes and downstream phosphorylation and ubiquitylation. Mol Syst Biol 11: 810-822. doi:10.15252/msb.20145880

Schilling B, Rardin MJ, MacLean BX, Zawadzka AM, Frewen BE, Cusack MP, Sorensen DJ, Bereman MS, Jing E, Wu CC, et al (2012) Platformindependent and label-free quantitation of proteomic data using MS1 extracted ion chromatograms in skyline: Application to protein 
acetylation and phosphorylation. Mol Cell Proteomics 11: 202-214. doi:10.1074/mcp.M112.017707

Schulte RJ, Campbell MA, Fischer WH, Sefton BM (1992) Tyrosine phosphorylation of CD22 during B cell activation. Science 258: 1001-1004. doi:10.1126/science.1279802

Schulz R, Marchenko ND, Holembowski L, Fingerle-Rowson G, Pesic M, Zender L, Dobbelstein M, Moll UM (2012) Inhibiting the HSP90 chaperone destabilizes macrophage migration inhibitory factor and thereby inhibits breast tumor progression. J Exp Med 209: 275-289. doi:10.1084/jem.20111117

Schwarz JJ, Wiese H, Tölle RC, Zarei M, Dengjel J, Warscheid B, Thedieck K (2015) Functional proteomics identifies acinus $L$ as a direct insulinand amino acid-dependent mammalian target of rapamycin complex 1 (mTORC1) substrate. Mol Cell Proteomics 14: 2042-2055. doi:10.1074/ mcp.M114.045807

Shannon P, Markiel A, Ozier O, Baliga NS, Wang JT, Ramage D, Amin N, Schwikowski B, Ideker T (2003) Cytoscape: A software environment for integrated models of biomolecular interaction networks. Genome Res 13: 2498-2504. doi:10.1101/gr.1239303

Siegers GM, Yang J, Duerr CU, Nielsen PJ, Reth M, Schamel WW (2006) Identification of disulfide bonds in the Ig-alpha/Ig-beta component of the B cell antigen receptor using the Drosophila S2 cell reconstitution system. Int Immunol 18: 1385-1396. doi:10.1093/ intimm/dxl072

Stuible M, Abella JV, Feldhammer M, Nossov M, Sangwan V, Blagoev B, Park M, Tremblay ML (2010) PTP1B targets the endosomal sorting machinery: Dephosphorylation of regulatory sites on the endosomal sorting complex required for transport component STAM2. J Biol Chem 285: 23899-23907. doi:10.1074/jbc.M110.115295

Tanno H, Yamaguchi T, Goto E, Ishido S, Komada M (2012) The Ankrd 13 family of UIM-bearing proteins regulates EGF receptor endocytosis from the plasma membrane. Mol Biol Cell 23: 1343-1353. doi:10.1091/mbc.E1109-0817

Tew KD, Manevich Y, Grek C, Xiong Y, Uys J, Townsend DM (2011) The role of glutathione S-transferase $P$ in signaling pathways and $S$ glutathionylation in cancer. Free Radic Biol Med 51: 299-313. doi:10.1016/j.freeradbiomed.2011.04.013

Tiganis T, Bennett AM (2007) Protein tyrosine phosphatase function: The substrate perspective. Biochem J 402: 1-15. doi:10.1042/BJ20061548

Tyanova S, Temu T, Sinitcyn P, Carlson A, Hein MY, Geiger T, Mann M, Cox J (2016) The Perseus computational platform for comprehensive analysis of (prote)omics data. Nat Methods 13: 731-740. doi:10.1038/ nmeth.3901

Vanshylla K, Bartsch C, Hitzing C, Krümpelmann L, Wienands J, Engels N (2018) Grb2 and GRAP connect the B cell antigen receptor to Erk MAP kinase activation in human B cells. Sci Rep 8: 4244. doi:10.1038/s41598-01822544-X

Wagner GP, Kin K, Lynch VJ (2012) Measurement of mRNA abundance using RNA-seq data: RPKM measure is inconsistent among samples. Theory Biosci 131: 281-285. doi:10.1007/s12064-012-0162-3

Wiśniewski JR, Hein MY, Cox J, Mann M (2014) A "proteomic ruler" for protein copy number and concentration estimation without spike-in standards. Mol Cell Proteomics 13: 3497-3506. doi:10.1074/ mcp.M113.037309

Xu H, An H, Hou J, Han C, Wang P, Yu Y, Cao X (2008) Phosphatase PTP1B negatively regulates MyD88- and TRIF-dependent proinflammatory cytokine and type I interferon production in TLR-triggered macrophages. Mol Immunol 45: 3545-3552. doi:10.1016/ j.molimm.2008.05.006

Yamanashi Y, Okada M, Semba T, Yamori T, Umemori H, Tsunasawa S, Toyoshima K, Kitamura D, Watanabe T, Yamamoto T (1993) Identification of HS1 protein as a major substrate of protein-tyrosine kinase(s) upon B-cell antigen receptor-mediated signaling. Proc Natl Acad Sci U S A 90: 3631-3635. doi:10.1073/pnas.90.8.3631

Yip SC, Saha S, Chernoff J (2010) PTP1B: A double agent in metabolism and oncogenesis. Trends Biochem Sci 35: 442-449. doi:10.1016/ j.tibs.2010.03.004

Yohannan J, Wienands J, Coggeshall KM, Justement LB (1999) Analysis of tyrosine phosphorylation-dependent interactions between stimulatory effector proteins and the B cell co-receptor CD22. J Biol Chem 274: 18769-18776. doi:10.1074/jbc.274.26.18769

Zhu JW, Brdicka T, Katsumoto TR, Lin J, Weiss A (2008) Structurally distinct phosphatases CD45 and CD148 both regulate B cell and macrophage immunoreceptor signaling. Immunity 28: 183-196. doi:10.1016/ j.immuni.2007.11.024

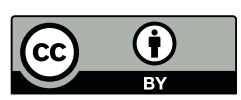

License: This article is available under a Creative Commons License (Attribution 4.0 International, as described at https://creativecommons.org/ licenses/by/4.0/). 